\title{
On Resolutions of Cyclic Quotient Singularities
}

\author{
By
}

\author{
Akira FujIKI
}

\section{Introduction}

Let $G$ be a finite cyclic group with a fixed generator $g$, acting on a complex affine $m$-space $\boldsymbol{C}^{m}=\boldsymbol{C}^{m}\left(z_{1}, \ldots, z_{m}\right)$ by the formula;

$$
g:\left(z_{1}, \ldots, z_{m}\right) \longrightarrow\left(e_{n}^{p_{1}} z_{1}, \ldots, e_{n}^{p_{m}} z_{m}\right)
$$

where $n, p_{i}, 1 \leqq i \leqq m, \quad$ are integers satisfying $0 \leqq p_{i}<n$ and $e_{n}^{p_{i}}=$ $\exp \left(2 \pi \sqrt{-1} p_{i} / n\right)$. Then the quotient space $X=C^{m} / G$ has the natural structure of a normal affine algebraic variety such that the quotient map $\pi: \boldsymbol{C}^{m} \rightarrow X$ is a morphism of algebraic varieties [10] [13]. We call this $X$ a cyclic quotient singularity and denote it often by $N_{n, p_{1}, \ldots, p_{m}}$ according to the particular expression of the generator $g$ as above.

Now the main purpose of this paper is to show the existence of certain natural ways of resolution of these cyclic quotient singularities, which have some good properties. (For the precise statement, see Theorem 1.) Such resolutions were first constructed by Hirzebruch in [3] when $m=2$ and then, by Ueno [12], when $m=3, p_{1}=1$ and $p_{2}=p_{3}$ in (1). On the other hand, the author recently learned that Mumford has found the equivariant resolutions of toroidal singularities, which contain cyclic quotient singularities as special cases [6]. However, our method is different from his and is connected more closely with the above expression of $g$. So it may be of some interest to compare the resolutions obtained here with those in [6].

In $\S 1$ we prove Theorem 1 and then, in $\S 2$ we apply this theorem to obtain resolutions of the general isolated quotient singularities and the isolated singularity with $C^{*}$ action in the case where the dimension 
of the spaces is 3. This is done by methods similar to those used in [1] for the former and in [8] for the latter respectively in the 2-dimensional cases. The unpleasant restriction on the dimension comes from the fact that we cannot prove the statement corresponding to Lemma 7 in higher dimensional cases.

In this paper the variety means an irreducible algebraic variety defined over $\boldsymbol{C}$ in the sense of FAC. Further we adopt the following notational conventions; for positive integers $n_{1}, \ldots, n_{l},\left(n_{1}, \ldots, n_{l}\right)$ denotes the greatest common divisor of them. If $T$ is an automorphism of $\boldsymbol{C}^{m}$ defined by $T\left(z_{1}, \ldots, z_{m}\right)=\left(\tau_{1} z_{1}, \ldots, \tau_{m} z_{m}\right)$ with $\tau_{i} \in C^{*}$, then we abbreviate it to $T=\left(\tau_{1}, \ldots, \tau_{m}\right)$. Moreover for $0 \leqq p<n, \quad e_{n}^{p}=\exp (2 \pi \sqrt{-1} p / n)$. $N_{n, p_{1}, \ldots, p_{m}}=\boldsymbol{C}^{m} /\{g\}$ if $g=\left(e_{n}^{p_{1}}, \ldots, e_{n}^{p_{m}}\right)$ in the above notation. We also use the notation $N_{n, p, q}$ to express $N_{n, 1, p, q}$ in 1.4.2.

In concluding the introduction, the author wishes to express his hearty thanks to Prof. S. Nakano and Dr. K. Ueno for their kind encouragement during the preparation of this paper.

\section{§1. Resolutions of Cyclic Quotient Singularities}

1.1. Let the notations be the same as in the introduction, except that in the sequel we assume $\left(n, p_{1}, \ldots, p_{m}\right)=1$ in the expression (1) of $g$. In this section we study the singular locus $S$ of $X$. For this purpose, let $F$ be the fixed point set of $G$ in $C^{m}$, namely, the set of those points whose stabilizers are nontrivial with respect to the action of $G$. This $F$, with reduced structure, is in general a subvariety of $\boldsymbol{C}^{m}$. But to describe $F$ more closely, we put in general, for any nonempty subset $M=\left\{m_{1}, \ldots, m_{k}\right\} \subseteq\{1, \ldots, m\}, \quad H(M)=\left\{\left(z_{1}\right) \in C^{m} ; z_{m_{1}}=z_{m_{2}}=\cdots=0\right\}$ and call it a coordinate subspace of $\boldsymbol{C}^{\boldsymbol{m}}$ defined by $M$. Then we have

Lemma 1. Let $F(i)$ be the fixed point set of $g^{i}$ for $1 \leqq i<n$. Then each $F(i)$ coincides with some coordinate subspace $H(M)$ of $\mathbb{C}^{m}$. Conversely, a coordinate subspace $H(M)$ of $\boldsymbol{C}^{m}$ coincides with $F(i)$ for some $i$ if and only if the following condition is satisfied; if we put $d=d(M)=\left(n, p_{j_{1}}, \ldots, p_{j_{m-k}}\right)$, then $d \chi\left(n, p_{m_{t}}\right)$ for any $t$, where $M=$ $\left\{m_{1}, \ldots, m_{k}\right\}$ and $\left\{j_{1}, \ldots, j_{m-k}\right\}$ is the complementary set of $M$ in $\{1, \ldots$, $m\}$. 
Proof. Suppose $g^{i}=\left(e_{n}^{p_{1}{ }^{i}}, \ldots, e_{n}^{p_{m} i}\right)$ has $t=t(i)$ 1's as its component, say, on the $i_{1}, \ldots, i_{t}$ th places. Then a point $z=\left(z_{1}, \ldots, z_{m}\right)$ is fixed by $g^{i}$ if and only if $z_{j}=0$ for any $j \neq i_{\alpha}, 1 \leqq \alpha \leqq t$. Thus if we take $M$ to be the complementary set of $\left\{i_{1}, \ldots, i_{t}\right\}$, then $F(i)=H(M)$ and the first assertion is proved. Next, for the given $M$, let $n_{1}=n / d(M)$. Then $n_{1}$ is easily seen to be the least integer $\mu$ for which $g^{\mu}$ has 1 on the $i$-th component for every $i \notin M$. Thus we have only to show that $H(M)=$ $F\left(n_{1}\right)$ if and only if the above condition holds. But $H(M)=F\left(n_{1}\right)$ is equivalent to the fact that for any $j \in M$, the $j$-th component of $g^{n_{1}}$ is different from 1 , or equivalently, $\left(e_{n}^{p_{j}}\right)^{n_{1}}=e_{n_{j}^{\prime}}^{p_{j}^{\prime} n_{1}} \neq 1$, where $n_{j}^{\prime}=$ $n /\left(n, p_{j}\right)$ and $p_{j}^{\prime}=p_{j} /\left(n, p_{j}\right)$. Then, since $\left(n_{j}^{\prime}, p_{j}^{\prime}\right)=1$, this in turn is equivalent to the fact that $n_{j}^{\prime} \nmid n_{1}$, namely, $(n / d) /\left(n /\left(n, p_{j}\right)\right)=\left(n, p_{j}\right) / d$ is not an integer for $j \in M$. This proves the second assertion. Q.E.D.

In particular, if $\left(n, p_{t}\right)=1$ for some $t$, hyperplane $z_{t}=0$ contains every $F(i)$.

Now let $G(i)$ be the stabilizer of the coordinate hyperplane $H\{i\}$, $i=1, \ldots, m, g(i)$ a generator of $G(i)$ and $G_{0}$ be the subgroup of $G$ generated by $\{G(i)\}_{i=1, \ldots, m}$. Each $g(i)$ has the form $\left(1, \ldots, e_{n_{1}}, \ldots, 1\right)$ with $e_{n_{i}}$ on the $i$-th component and $n_{i} \mid n$. From this, we see that $C^{m}(z) / G_{0} \cong$ $\boldsymbol{C}^{m}(w)$ and in fact the quotient map $h_{0}: \boldsymbol{C}^{m}(z) \rightarrow \boldsymbol{C}^{m}(w)$ is defined by $(w)=h_{0}(z)=\left(z_{1}^{n}, \ldots, z_{m}^{n_{m}}\right)$. Further the quotient group $\bar{G}=G / G_{0}$ acts naturally on $\boldsymbol{C}^{m}(w)$ by $\bar{g}=\left(e_{n}^{p_{1} n_{1}}, \ldots, e_{n}^{p_{m} n_{m}}\right)$ so that $X=\boldsymbol{C}^{m}(w) / \bar{G}$, where $\bar{g}$ is the natural image of $g$ in $\bar{G}$. Then $\bar{G}$ has no element whose fixed point set is a coordinate hyperplane of $\boldsymbol{C}^{m}(w)$. For otherwise, there exists an element $\bar{g}^{k} \in \bar{G}$, the fixed point set of which is, say, $H\{1\}$ of $\boldsymbol{C}^{m}(w)$. Then this has the form $\bar{g}^{k}=\left(e_{t}, 1, \ldots, 1\right)$ and we infer from this that $g^{k}$ has the form $\left(e_{n}^{p_{1} k}, e_{n_{2}}^{t_{2}}, \ldots, e_{n_{m}}^{t_{n}}\right)$ and hence $g^{k} g(2)^{-t_{2}} \ldots g(m)^{-t_{m}}=$ $\left(e_{n}^{p_{1} k}, 1, \ldots, 1\right) \in G(1), g^{k} \in G_{0}$. This implies $\bar{g}^{k}=e$, the unit element of $G$, which is a contradiction. Now we say that a cyclic group acting on $\boldsymbol{C}^{m}$ as above is small, if it has no element whose fixed point set coincides with some coordinate hyperplane (c.f. [9]). Thus we have shown that in the expression $X=C^{m} / G$ of the cyclic quotient singularities, we may always take $G$ to be small.

The following lemma is a special case of the fundamental result of Prill [9, Theorem 2]. 
Lemma 2. Two cyclic quotient singularities $N_{n, p_{1}, \ldots p_{m}}=C^{m} / G$ and $N_{n, p_{1}^{\prime}, \ldots, p_{m}^{\prime}}=\mathbb{C}^{m} / G^{\prime}$, with $G$ and $G^{\prime}$ small, are isomorphic if and only if $n=n^{\prime}$, and there exist a permutation $\tau$ of $\{1, \ldots, m\}$ and an integer $k, 0<k<m$, such that $(k, n)=1$ and $p_{i} k \equiv p_{\pi(i)}^{\prime} \bmod n$.

In fact, Prill has proved more precisely that $\mathbb{C}^{m} / G$ and $\mathbb{C}^{m} / G^{\prime}$ are analytically isomorphic as germs of analytic spaces at $\pi(0)$ and $\pi^{\prime}(0)$ respectively, if and only if $G$ and $G^{\prime}$ are conjugate in $G L(m, \boldsymbol{C})$, where $\pi$ and $\pi^{\prime}$ are quotient maps.

From this we get the following.

Corollary. If $G$ is small, then the singular locus $S$ of $X$ coincides with $\pi(F)$, where $F$ is the fixed point set of $G$.

Proof. It is clear that $S \subseteq \pi(F)$. So let $\mathfrak{P} \in \pi(F)$ and take a point $\mathfrak{Q} \in F$ such that $\pi(\mathfrak{Q})=\mathfrak{B}$. Then there exists a neighborhood $U$ of $\mathfrak{Q}$ in $\mathbb{C}^{m}$ such that if $G_{\mathfrak{Q}}$ is the stabilizer of $\mathfrak{Q}$, then $U$ is $G_{\mathfrak{Q}}$ invariant and the quotient $U / G_{Q}$ is analytically isomorphic to some neighborhood $V$ of $\mathfrak{B}$. On the other hand, as we have already seen, the fixed point set of $g_{\mathfrak{D}}$, a generator of $G_{\mathfrak{D}}$, is some coordinate subspace $H(M)$ of $\mathbb{C}^{\boldsymbol{m}}$. After a suitable renumbering of coordinates if necessary, we may assume that $M=\{1, \ldots, k\}$, where $\operatorname{dim} H(M)=m-k$ and that $g_{\Sigma_{0}}$ acts on $C^{k}$ by $g_{\mathfrak{Q}}=\left(e_{n_{0}}^{s_{1}}, \ldots, e_{n_{0}}^{s_{k}}\right)$, where $s_{i}$ are some integers. Let $\mathfrak{Q}^{\prime}=\left(0, z^{\prime}\right) \in$ $\mathbb{C}^{k} / G_{\mathfrak{Q}} \times \boldsymbol{C}^{m-k}$ be the image of $\mathfrak{Q}$ by the quotient map $\mathbb{C}^{m} \rightarrow \mathbb{C}^{k} / G_{\mathfrak{Q}} \times$ $\boldsymbol{C}^{m-k}$ composed with the above isomorphism. Then $\left(U / G_{\mathfrak{Q}}, \pi(\mathfrak{Q})\right)$ and $\left(\boldsymbol{C}^{k} / G_{\mathfrak{Q}} \times \boldsymbol{C}^{m-k}, \mathfrak{Q}^{\prime}\right)$ must be analytically isomorphic as germs of analytic spaces. Then if $V$ is nonsingular, we conclude that $\boldsymbol{C}^{k} / \boldsymbol{G}_{\mathfrak{D}} \times \boldsymbol{C}^{m-k}$ and hence $C^{k} / G_{\emptyset}$ is nonsingular. But since it is easy to see that $G_{\emptyset}$, considered naturally as a subgroup of $G L(k, C)$, is small, we have $G_{\mathfrak{Q}}=\{e\}$ by the lemma. This contradicts the assumption that $\mathfrak{Q} \in F$.

Q.E.D.

Remark 1. By Lemma 1 we have in particular

a) $G$ is small if and only if $\left(n, p_{1}, \ldots, \hat{p}_{i}, \ldots, p_{m}\right)=1$ for every $i$, where $\hat{p}_{i}$ means that $p_{i}$ is omitted, and

b) if $G$ is small, then $\operatorname{dim} S=0$ if and only if $\left(n, p_{i}\right)=1$ for every $i$. 
In fact, $a$ ) and the sufficiency of the condition in $b$ ) are immediate. So suppose $\operatorname{dim} S=0$, and $\left(n, p_{i}\right)>1$ for some $i$. Let $\left\{j_{1}, \ldots, j_{k}\right\}$ be the subset of $\{1, \ldots, m\}$ consisting of those elements for which $\left(n, p_{j_{\alpha}}\right)$ is divisible by $\left(n, p_{i}\right)$ for every $\alpha, 1 \leqq \alpha \leqq k$, and let $M$ be the complementary set of $\left\{j_{1}, \ldots, j_{k}\right\}$ in $\{1, \ldots, m\}$. Then by Lemma $1, H(M)$ coincides with $F(i)$ for some $i$. But since $F(i)=\{0\}$ by the assumption, $M$ must coincide with $\{1, \ldots, m\}$. This is a contradiction because $i \notin M$.

1.2. The next procedure is the key step in the course of our resolution of $X$. Namely, we propose to show the following

Lemma 3. Suppose $X=N_{n, p_{1} \ldots, p_{m}}$ is a cyclic quotient singularity and assume that the group $G$ is small. Then there exist a variety $X_{1}$, a finite affine open covering $\mathfrak{U}=\left\{U_{1}, \ldots, U_{l}\right\}$ of $X_{1}$ for an integer $l, 1 \leqq l \leqq m$, and a proper birational morphism $f: X_{1} \rightarrow X$ such that for each $i$ there are isomorphisms $\varphi_{i}: U_{i} \cong N_{p_{i}^{\prime}, q_{1}^{i}, \ldots . q_{m}^{2}}$, where the integers $p_{i}^{\prime}$ and $q_{\alpha}^{i}$ are determined by the following formula:

$$
\left\{\begin{array}{l}
p_{i}^{\prime}=p_{i} / d, \quad \text { with } \quad d=\left(p_{1}, \ldots, p_{m}\right), \\
q_{\alpha}^{i} \equiv p_{\alpha}^{\prime} \quad \bmod p_{i}^{\prime} \text { if } \alpha \neq i, \text { and } 0 \leqq q_{\alpha}^{i}<p_{i}^{\prime} \\
q_{i}^{i}+n \equiv 0 \quad \bmod p_{i}^{\prime} .
\end{array}\right.
$$

Proof. The construction we have in mind when $p_{i}>0$ for every $i$ is roughly as follows; first, we take an abelian covering $h: \mathbb{C}^{m}(t) \rightarrow$ $C^{m}(z)$ with the covering transformation group $H$ and then, define an action of $G$ on $\mathbb{C}^{m}(t)$ compatible with $h$ so that $X=\mathbb{C}^{m}(t) / G \oplus H$. Next we perform a monoidal transformation $\sigma: W_{0} \rightarrow \mathbb{C}^{m}(t)$ at the origin and observe that the actions of $G$ and $H$ extend naturally onto $W_{0}$. Finally, we put $X_{1}=W_{0} / G \oplus H$ and define $f$ to be the morphism induced by $\sigma$. Then we see that this $X_{1}$ and $f$ have the desired properties.

Now we shall see these more closely. First we consider the case where $p_{i}>0$ for each $i$. Then the abelian covering $h: \mathbb{C}^{m}(t) \rightarrow \mathbb{C}^{m}(z)$ is defined by

$$
h\left(t_{1}, \ldots, t_{m}\right)=\left(t_{1}^{p_{1}}, \ldots, t_{m}^{p_{m}}\right)
$$

The covering transformation group $H$ of $h$ is isomorphic to the direct 
sum $G_{1} \oplus \cdots \oplus G_{m}$, where $G_{i}$ is the cyclic group of order $p_{i}$, a generator $g_{i}$ of which acts on $C^{m}(t)$ by $g_{i}=\left(1, \ldots, e_{p_{i}}, \ldots, 1\right)$ with $e_{p_{i}}$ on the $i$-th place. Moreover, we define the action of $g$ on $C^{m}(t)$ by

$$
g=\left(e_{n}, \ldots, e_{n}\right) .
$$

Then it is easy to see that this is compatible with $h$, namely, the equality $g \cdot h=h \cdot g$ holds. Now let $\sigma: W_{0} \rightarrow C^{m}(t)$ be the monoidal transformation at the origin. Then $W_{0}$ has the natural structure of a line bundle over a projective $(m-1)$-space $\boldsymbol{P}^{m-1}$ with homogeneous coordinates $\left(\zeta_{1}: \cdots\right.$ : $\left.\xi_{m}\right)$. Indeed, if we define $V_{i}=\left\{(\xi) \in \mathbb{P}^{m-1} ; \xi_{i} \neq 0\right\}$, then $W_{0}$ is expressed as $W_{0}=\bigcup_{i-1}^{m}\left(V_{i} \times \boldsymbol{C}\right)$, where $\left(p, \zeta_{i}\right) \in V_{i} \times \boldsymbol{C}$ and $\left(q, \zeta_{j}\right) \in V_{j} \times \boldsymbol{C}$ are identified if and only if $p=q$ and $\zeta_{i}=\left(\xi_{i} / \xi_{j}\right) \zeta_{j}$. Then the map $\sigma$ has the following form on each $W_{i}^{0}=V_{i} \times C$;

$$
\left\{\begin{array}{l}
t_{j}=\left(\xi_{j} / \zeta_{i}\right) \zeta_{i} \quad \text { if } \quad j \neq i \\
t_{i}=\zeta_{i}
\end{array}\right.
$$

Note that $W_{i}^{0}$ is isomorphic to $\mathbb{C}^{m}$ with the coordinates $\left(\xi_{1} / \xi_{i}, \ldots, \xi_{m} / \xi_{i}\right.$, $\zeta_{i}$ ). By (3) we see that the actions of $g$ and $g_{i}, i=1, \ldots, m$, extend onto $W_{0}$ as follows; on $W_{i}^{0}$,

$$
\left\{\begin{array}{l}
g_{j}=\left(1, \ldots, 1, e_{p_{j}}, 1, \ldots, 1\right) \text { with } e_{p_{j}} \text { on the } j \text {-th place, if } j \neq i \\
g=\left(1, \ldots, 1, e_{n}\right) \\
g_{i}=\left(e_{p_{i}}^{-1}, \ldots, e_{p_{i}}^{-1}, e_{p_{i}}\right) .
\end{array}\right.
$$

Then, as in 1.1 if we set $W_{i}^{1}=W_{i}^{0} / \check{G}_{i}$ with $\check{G}_{i}=G \oplus G_{1} \oplus \cdots \cdots \oplus \hat{G}_{i} \oplus \cdots \cdots$ $\oplus G_{m}$, then $W_{i}^{1} \cong \boldsymbol{C}^{m}\left(w_{1}^{i 1}, \ldots, w_{m}^{i 1}\right)$ and if we identify $W_{i}^{1}$ with $\boldsymbol{C}^{m}\left(w^{i 1}\right)$ by this isomorphism, then the quotient map is defined by

$$
\rho_{i}:\left(\xi_{1} / \xi_{i}, \ldots, \xi_{m} / \xi_{i}, \zeta_{i}\right) \longrightarrow\left(\left(\xi_{1} / \xi_{i}\right)^{p_{1}}, \ldots, \zeta_{i}^{n}, \ldots,\left(\xi_{m} / \xi_{i}\right)^{p_{m}}\right)
$$

with $\zeta_{i}^{n}$ on the $i$-th place. Moreover the action of $G_{i}$ induced on $W_{i}^{1}$ takes the form;

$$
g_{i}=\left(e_{p_{i}}^{-p_{1}}, \ldots, e_{p_{i}}^{n}, \ldots, e_{p_{i}}^{-p_{m}}\right)
$$

However, $G_{i}$ may not be small with respect to the action on $W_{i}^{1}$. In 
fact, by Remark $1 a), G_{i}$ is not small if and only if $d=\left(p_{1}, \ldots, p_{m}\right)>1$, since $G$ is small. In any case we put $W_{i}=W_{i}^{1} /\left\{g_{i}^{d}\right\}$. Then again as in $1.1, W_{i} \cong \boldsymbol{C}^{m}\left(w_{1}^{i}, \ldots, w_{m}^{i}\right)$, the quotient map $\bar{\rho}_{i}: W_{i}^{1} \rightarrow W_{i}$ is defined by

$$
\bar{\rho}_{i}\left(w_{1}^{i 1}, \ldots, w_{m}^{i 1}\right)=\left(w_{1}^{i m}, \ldots,\left(w_{i}^{i 1}\right)^{d}, \ldots, w_{m}^{i 1}\right),
$$

and the induced action of $\bar{G}_{i}=G_{i} /\left\{g_{i}^{d}\right\}$ has the form

$$
\bar{g}_{i}=\left(e^{-p_{i}^{\prime} p_{1}^{\prime}}, \ldots, e_{p_{i}}^{n \prime}, \ldots, e_{p_{i}^{\prime}}^{-p_{m}^{\prime}}\right),
$$

where $p_{i}^{\prime}=p_{i} / d, \bar{g}_{i}$ is the natural image of $g_{i}$ in $\bar{G}_{i}$, and $\bar{G}_{i}$ is small. Now we set $X_{1}=W_{0} / G \oplus H$ and $U_{\imath}=\pi\left(W_{i}^{0}\right), 1 \leqq i \leqq m$, where $\pi: W_{0} \rightarrow X_{1}$ is the quotient map. Then by the above description we have $U_{i}=$ $W_{i} / \bar{G}_{i}$, and hence $\mathfrak{U}=\left\{U_{1}, \ldots, U_{m}\right\}$ makes a finite affine open covering of $X_{1}$ such that each member $U_{i}$ is isomorphic to a cyclic quotient singularity $N_{p_{i}^{\prime}, q_{1}^{i}, \ldots, q_{m}^{2}}$ of order $p_{i}^{\prime}$. Here the integers $q_{\alpha}^{i}$ are defined by (2), taking $\bar{g}_{i}^{-1}$ as a generator of $\bar{G}_{i}$. Finally, if we recall that $X=$ $\boldsymbol{C}^{m}(z) / G \oplus H$, and that the action of $G$ and $H$ on $W_{0}$ and $X$ commute with $\sigma$, then we see that $\sigma$ induces a birational morphism $f: X_{1} \rightarrow X$.

Next, we consider the case when $p_{i}=0$ for some $i$. After a suitable permutation of $w_{i}$, we may assume that $p_{l+1}=\cdots=p_{m}=0$ and $p_{i}>0$ for $i \leqq l$ for some $l>0$. Then $g=\left(e_{n}^{p_{1}}, \ldots, e_{n}^{p_{1}}, 1, \ldots, 1\right)$ and $X$ is naturally isomorphic to $\left(\boldsymbol{C}^{l} / G\right) \times \boldsymbol{C}^{m-l}$, where the action of $G$ on $\boldsymbol{C}^{l}$ is defined by $g=\left(e_{n}^{p_{1}}, \ldots, e_{n}^{p_{l}}\right)$. Put $Y=\boldsymbol{C}^{l} / G$. Then we may apply the above considerations to $Y$ instead of to $X$. Suppose $Y_{1}, \mathfrak{B}=\left\{V_{1}, \ldots, V_{l}\right\}$, and $g: Y_{1} \rightarrow Y$ correspond in the above consideration to $X_{1}, \mathfrak{U}$, and $f: X_{1} \rightarrow X$ respectively. Then we put $X_{1}=Y \times \mathbb{C}^{m-l}, \mathfrak{U}=\left\{U_{i} ; U_{i}=V_{i} \times \boldsymbol{C}, 1 \leqq i \leqq l\right\}$, and $f=g \times i d: X_{1} \rightarrow X$, where id is the identity map of $\boldsymbol{C}^{m-l}$. Correspondingly, we get the groups $\bar{G}_{i}$ and the isomorphisms $\varphi_{i}: U_{i} \Im$ $N_{p_{i}^{\prime}, q_{1}, \ldots, q_{m}}$ with $q_{i}=0$ for $i \geqq l+1$. The relations in (2) are obvious, and hence the proof of the lemma is completed.

Now we summarize in the following lemma the properties of the covering $\mathfrak{H}$, and the morphism $f$, thus obtained.

Lemma 4. i) If we denote by $\pi_{i}: W_{i} \rightarrow U_{i}, 1 \leqq i \leqq l$, the quotient maps, then $W_{i j}=\pi_{i}^{-1}\left(U_{i} \cap U_{j}\right)$ is an open subset of $W_{i}$ defined by $w_{j}^{i} \neq 0$.

ii) The multivalued map $\pi_{i, j}=\pi_{i}^{-1} \pi_{j}: \pi_{. j}^{-1}\left(U_{i} \cap U_{i}\right) \rightarrow \pi_{i}^{-1}\left(U_{i} \cap U_{j}\right)$ is de- 
fined by

$$
\left\{\begin{array}{l}
w_{k}^{i}=w_{k}^{i}\left(w_{j}^{i}\right)^{-p_{k}^{\prime} / p_{i}^{\prime}} \quad \text { if } k \neq i, \neq j \\
w_{i}^{i}=w_{j}^{j}\left(w_{i}^{j}\right)^{n / p_{i}^{\prime}} \\
w_{j}^{i}=\left(w_{i}^{j}\right)^{-p_{j}^{\prime} / p_{i}^{\prime}}
\end{array}\right.
$$

where $\left(w_{i}^{j}\right)^{i / p^{\prime}}$ denotes any $p_{i}^{\prime}$-th root of $w_{i}^{j}$.

iii) The multivalued map $\pi_{0 i}=\pi^{-1} \pi_{i}: W_{i} \rightarrow C^{m}(z)$ is defined by

$$
\left\{\begin{array}{l}
z_{k}=w_{k}^{i}\left(w_{i}^{i}\right)^{p_{k}^{\prime} / n} \quad \text { if } k \neq i \\
z_{i}=\left(w_{i}^{i}\right)^{p_{i}^{\prime} / n}
\end{array}\right.
$$

iv) Let $T$ be the automorphism of $X$ induced by an automorphism $T^{\prime}$ of $\mathbb{C}^{m}(z)$ of the form $T^{\prime}=\left(\tau_{1}, \ldots, \tau_{m}\right)$, then $T$ extends uniquely onto $X$ so that it leaves each $U_{i}$ invariant and $T \mid U_{i}$ is induced by the automorphism $T_{i}$ of $W_{i}=\boldsymbol{C}^{m}\left(w^{i}\right)$ of the form

$$
T_{i}=\left(\tau_{1} \tau_{i}^{-p_{1} / p_{i}}, \ldots, \tau_{i}^{n / p_{1}}, \ldots, \tau_{m} \tau_{i}^{-p_{m} / p_{\imath}}\right)
$$

v) Let $F_{0}$ be the fixed point set of $g, S_{0}=\pi\left(F_{0}\right)$, and $S_{1}=f^{-1}\left(S_{0}\right)$, then $\pi_{i}^{-1}\left(U_{i} \cap S_{1}\right)$ is defined by $w_{i}^{i}=0$ in $W_{i}$.

The proofs are all straightforward and we omit them. We only note that the construction of $X_{1}$ and $f_{1}$ depends crucially on the choice of a generator $g$ of $G$ as in (1), or equivalently, on the choice of an isomorphism $\varphi: X \simeq N_{n, p_{1}, \ldots, p_{m}}$ (see an example in 1.4).

1.3. For resolutions of the cyclic quotient singularities, we have to deal with a little more general situation. So let $X$ be a variety and suppose there exists a finite affine open covering $\mathfrak{U}=\left\{U_{1}, \ldots, U_{s}\right\}$ of $X$ such that there exists for each $i$ an isomorphism $\varphi_{i}: U_{i} \cong N_{n_{i} p_{1}^{(i)} \ldots p_{m}^{(i)}}$, where each $N_{n_{i} p_{1}^{(1)} \ldots p_{m}^{(i)}}=C^{m}\left(u_{1}^{i}, \ldots, u_{m}^{i}\right) / G_{i}$ is a cyclic quotient singularity defined in the introduction. In this case we call $n_{i}$ the order of $U_{i}$. We denote such $X$ and $\mathfrak{U}$ simply by the pair $(X, \mathfrak{U})$. Hence, when we speak of a pair $(X, \mathfrak{H})$, it is supposed that we are given a variety $X$ and a finite affine open covering $\mathfrak{U}$ of $X$ such that to each $U_{i}$ there 
are assoicated an $m$-dimensional affine space $\boldsymbol{C}^{m}$ with the coordinate system $\left(u_{1}^{i}, \ldots, u_{m}^{i}\right)$, a cyclic group $G_{i}$ with a fixed generator $g_{i}$, and an action of $G_{i}$ of the form $g_{i}=\left(e_{n i}^{p_{1}^{(i)}}, \ldots, e_{n_{i}}^{p_{n}^{(1)}}\right)$ on $\mathbb{C}^{m}$ together with an isomorphism $\varphi_{i}: U_{i} \simeq C^{m} / G_{i}$. Now let $(X, \mathfrak{u})$ be a pair. We say that $U_{i} \in \mathfrak{U}$ is adjacent to $U_{j} \in \mathfrak{U}$ if $\pi_{i}^{-1}\left(U_{i} \cap U_{j}\right)$ is defined by the equation $u_{k}^{i} \neq 0$ for some $k=k(i, j)$. Note that in this case $U_{j}$ is also adjacent to $U_{i}$.

Definition 1. A pair $(X, \mathfrak{l})$ as above is said to be admissible if the following three conditions are satisfied:

( $\alpha$ ) Any two members $U_{i}$ and $U_{j}$ of $\mathfrak{U}$ can be connected by a finite sequence of adjacent ones, namely, there exists a finite sequence $U_{1}, \ldots, U_{d}$ with $U_{\alpha} \in \mathfrak{U}$ such that $U_{1}=U_{i}, U_{d}=U_{j}$ and $U_{t-1}$ is adjacent to $U_{t}$ for $0<t \leqq d$.

( $\beta$ ) Suppose $U_{i}$ is adjacent to $U_{j}$ and $\pi_{i}^{-1}\left(U_{i} \cap U_{j}\right)$ (resp. $\pi_{j}^{-1}\left(U_{i} \subset\right.$ $\left.U_{j}\right)$ ) is defined by $u_{k}^{i} \neq 0$ (resp. $u_{k^{\prime}}^{j} \neq 0$ ) in $\mathbb{C}^{m}\left(u^{i}\right)$ (resp. in $\boldsymbol{C}^{m}\left(u^{j}\right)$ ), where $\pi_{i}: \boldsymbol{C}^{m} \rightarrow U_{i} \quad$ (resp. $\pi_{j}: \boldsymbol{C}^{m} \rightarrow U_{j}$ ) is the quotient map $\rho_{i}: \boldsymbol{C}^{m} \rightarrow$ $\mathbb{C}^{m} / G_{i}$ (resp. $\rho_{j}: \mathbb{C}^{m} \rightarrow \mathbb{C}^{m} / G_{j}$ ) composed with the isomorphism $\varphi_{i}^{-1}$ (resp. $\left.\varphi_{j}^{-1}\right)$. Then the multivalued map $\pi_{i j}=\pi_{i}^{-1} \pi_{j}: \pi_{j}^{-1}\left(U_{i} \cap U_{j}\right) \rightarrow \pi_{i}^{-1}\left(U_{i}\right.$ $\cap U_{j}$ ) has the following form;

$$
\left\{\begin{array}{l}
u_{k}^{i}=\left(u_{l}^{j}\right)^{-n_{J} / n_{\imath}} \\
u_{s}^{i}=\left(u_{l}^{j}\right)^{a(s)} u_{\pi(s)}^{j} \quad \text { if } s \neq k,
\end{array}\right.
$$

where $n_{i}=\operatorname{ord} U_{i}, n_{j}=\operatorname{ord} U_{j}, a(s), 1 \leqq s \leqq m, s \neq k$, are certain rational numbers, and finally $\pi:\{1, \ldots, \hat{k}, \ldots, m\} \rightarrow\{1, \ldots, \hat{l}, \ldots, m\}$ is some bijective map.

( $\gamma$ ) The groups $G_{i}$ are all small.

Remark 2. a) For any point $P \in \mathbb{C}^{m}\left(u^{i}\right) \pi_{i}^{-1}(\pi(P))$ consists of at most $n_{i}$ distinct points. From this, we see that the denominators of $a(s)$ do not exceed $n_{i}$ in their irreducible expressions.

b) Let $U_{i}$ and $U_{j}$ be as in $(\beta)$ and if $\pi_{i j}$ has the form stated in $(\beta)$, then so does $\pi_{j i}$ as is seen by solving the equation with respect to $u^{j}$.

Now let $(X, \mathfrak{U})$ and $(Y, \mathfrak{B})$ be admissible pairs and $f: X \rightarrow Y$ be a proper birational morphism. We say that $f$ is compatible with the 
coverings $\mathfrak{U}$ and $\mathfrak{B}$ if for any $V_{\alpha} \in \mathfrak{B}, \mathfrak{I}_{\alpha}=\left\{U_{i} \in \mathfrak{U} \mid U_{i} \subseteq f^{-1}\left(V_{\alpha}\right)\right\}$ makes an affine open covering of $f^{-1}\left(V_{\alpha}\right)$. Then the pair $\left(f^{-1}\left(V_{\alpha}\right), \mathfrak{H}_{\alpha}\right)$ with groups $G_{i}$, coordinates $\left\{u^{i}\right\}$, and isomorphisms $\varphi_{i}: U_{i} \cong C^{m}\left(u^{i}\right) / G_{i}$ induced by those of the original $U_{i}$ is admissible. We denote this also by $f^{-1}\left(\left(V_{\alpha},\left\{V_{\alpha}\right\}\right)\right)$.

Definition 2. Suppose $(X, \mathfrak{U}),(Y, \mathfrak{B})$, and $f$ are as above. We say that $f$ is admissible as a morphism of admissible pairs $(X, \mathfrak{i n})$ and $(Y, \mathfrak{B})$ if $f$ satisfies the following two conditions:

$(\alpha) \quad f$ is compatible with the coverings $\mathfrak{U}$ and $\mathfrak{B}$.

$(\beta)$ Suppose $f\left(U_{i}\right) \subseteq V_{\alpha}$, then the multivalued map $\pi_{\alpha}^{-1} f \pi_{i}: \boldsymbol{C}^{m}\left(u^{i}\right) \rightarrow$ $C^{m}\left(v^{\alpha}\right)$ takes the following form;

$$
v_{s}^{\alpha}=\left(u_{1}^{i}\right)^{b(1, s)} \cdots\left(u_{m}^{i}\right)^{b(m, s)}, \quad 1 \leqq s \leqq m,
$$

where $b(i, s)$ are positive and rational numbers. Moreover an automorphism $T$ of $X$ is said to be admissible if $T\left(U_{i}\right)=U_{i}$ for every $U_{i} \in \mathfrak{l l}$ and $T \mid U_{i}$ is induced by an automorphism $\widetilde{T}_{i}$ of $\mathbb{C}^{m}\left(u^{i}\right)$ of the form $\widetilde{T}_{i}=\left(\tau_{1}, \ldots, \tau_{m}\right), \tau_{i} \in \boldsymbol{C}^{*}$, where $U_{i} \cong \boldsymbol{C}^{m}\left(u^{i}\right) / G_{i}$.

For example, let $X=C^{m} / G$ be a cyclic quotient singularity. Then $(X,\{X\})$ can be trivially regarded as an admissible pair. Next let $X_{1}$, $\mathfrak{l l}$, and $f: X_{1} \rightarrow X$ be as in Lemma 3 . Then, by Lemma $4\left(X_{1}, \mathfrak{U}\right)$ and $f$ are admissible, and any admissible automorphism of $X$ extends uniquely to that of $\left(X_{1}, \mathfrak{U}\right)$.

On the other hand, if $X$ is nonsingular in an admissible pair $(X, \mathfrak{n})$, then since $G_{i}$ are small, we must have $n_{i}=1$ and $U_{i}$ are isomorphic to $\boldsymbol{C}^{m}\left(u^{i}\right)$. Then the multivalued maps $\pi_{i j}$ in $(\beta)$ are nothing but the trandition functions with respect to this covering. Rewriting these, we have

$$
\left\{\begin{array}{l}
u_{k}^{i}=\left(u_{l}^{j}\right)^{-1} \\
u_{s}^{i}=\left(u_{l}^{j}\right)^{a(s)} u_{\pi(s)}^{i}, \quad s \neq k,
\end{array}\right.
$$

where $a(s)$ are now integers.

Now recall that a resolution of a variety $X$ is a pair $(\tilde{X}, f)$ consisting of a variety $\tilde{X}$ and a proper birational morphism $f: \tilde{X} \rightarrow X$ such that $f$ is isomorphic outside $f^{-1}(S), S$ being the singular locus of $X$. Then we are able to state our main theorem. 
Theorem 1. Suppose $(X, \mathfrak{l n})$ is an admissible pair. Then there exists a resolution $(\tilde{X}, f)$ of $X$ and a finite affine open covering $\tilde{\mathfrak{V}}$ of $\tilde{X}$ such that the following conditions are satisfied;

1) $(\tilde{X}, \tilde{\mathfrak{l}})$ is admissible,

2) $f$ is admissible as a morphism of admissible pairs $(\tilde{X}, \tilde{\mathfrak{l}})$ and $(X, \mathfrak{l l})$,

3) any admissible automorphism $T$ of $U_{i}$ extends uniquely to that of $f^{-1}\left(\left(U_{i},\left\{U_{i}\right\}\right)\right)$, and

4) $f^{-1}(S) \cap \tilde{U}_{\alpha}$ is defined by $\tilde{u}_{k_{1}}^{\alpha} \ldots \tilde{u}_{k_{t}}^{\alpha}=0$ in $\tilde{U}_{\alpha} \cong \boldsymbol{C}^{m}\left(\tilde{u}^{\alpha}\right)$ for some $k_{1}, \ldots, k_{t}$, if it is not empty.

From 4) we derive easily the following.

Corollary. Let $E=f^{-1}(S)$. Then $E$ has only normal crossings in $\tilde{X}$ and every irreducible component $E_{i}$ of $E$ is nonsingular and rational. Further $E_{i}$ is covered by finite affine open subsets, each of which is isomorphic to $\mathbf{C}^{\mathbf{m}-1}$.

Proof of Theorem 1. First we consider the set $\Phi$ of maps $\varphi: N \rightarrow$ $N \cup\{0\}$ such that $\varphi(N) \neq 0$ and $\varphi(n)=0$ for all but a finite number of $n$ 's, where $N$ is the set of natural numbers. We shall introduce an order on the set $\Phi$ in the following manner. Let $\varphi_{1}, \varphi_{2} \in \Phi$ and $n_{0}$ be the largest integer for which $\varphi_{1}\left(n_{0}\right) \neq \varphi_{2}\left(n_{0}\right)$. Then we define $\varphi_{1}<\varphi_{2}$ by the inequality $\varphi_{1}\left(n_{0}\right)<\varphi_{2}\left(n_{0}\right)$. By this, $\Phi$ becomes a totally ordered set with a minimal element $\varphi_{0}$, which is defined by $\varphi_{0}(1)=1$ and $\varphi_{0}(n)=0$ for $n \geqq 2$. We associate then to each admissible pair $(X, \mathfrak{U})$ an element of $\Phi$, which we denote simply by $\varphi_{X}$ since no confusion may arise, by $\varphi_{X}(n)=\#\left\{U_{i} \in \mathfrak{l} \mathfrak{l}\right.$; ord $\left.U_{i}=n\right\}$ for $n \in N$, where \# means the number of elements of the corresponding set. Then by the above remark we may try to prove the theorem by induction on $\varphi_{X}$. If $\varphi_{X}=\varphi_{0}$, or more generally, if $\varphi_{X}(n)=0$ for $n \geqq 2$, then it is sufficient to define $(\tilde{X}, \tilde{\mathfrak{u}})=$ $(X, \mathfrak{l l})$ and $f=i d_{X}$, the identity map of $X$, because then $X$ is nonsingular. Thus we may assume that $\varphi_{X}(n) \geqq 1$ for some $n \geqq 2$ and that the theorem has already been proved for admissible pairs $(Y, \mathfrak{B})$ for which $\varphi_{Y}<\varphi_{X}$.

We define an integer $l(i)$ for each $U_{i}$ by $l(i)=\#\left\{p_{k}^{(i)} ; p_{h}^{(i)} \neq 0,1 \leqq\right.$ 
$k \leqq m\}$, where $U_{i}$ is isomorphic to $N_{n_{1}, p_{1}^{(1)}, \ldots, p_{m}^{(1)}}$ by the isomorphism $\varphi_{i}$. Clearly this is equal to the codimension of the fixed point set of $G_{i}$, and hence does not depend on the particular choice of the generator. Set $l=\max _{i} l(i)$ and take and fix one $U_{i_{0}} \in U$ for which $l=l\left(i_{0}\right)$. Next, let $F_{0}$ be the fixed point set of $g_{i 0}, S_{i 0}=\pi_{i 0}\left(F_{0}\right)$ and $S_{0}$ the closure of $S_{i 0}$ in $X$, where $\pi_{i 0}: \mathbb{C}^{m} \rightarrow U_{i 0}$ is the quotient map. Then our purpose is to construct an admissible pair $\left(X_{1}, \mathfrak{U}_{1}\right)$ and a birational morphism $f_{1}: X_{1} \rightarrow X$, such that $f_{1}$ is admissible for $\left(X_{1}, \mathfrak{U}_{1}\right)$ and $(X, \mathfrak{u})$, that $f_{1}$ is isomorphic outside $f^{-1}\left(S_{0}\right)$, and that $\varphi_{X_{1}}<\varphi_{X}$. For this, first we construct for each $U_{i}$ an admissible pair $\left(W_{i}, \mathfrak{V}_{i}\right)$ and a birational morphism $\psi_{i}: W_{i} \rightarrow U_{i}$ admissible for $\left(W_{i}, \mathfrak{B}_{i}\right)$ and $\left(U_{i},\left\{U_{i}\right\}\right)$, such that $\psi_{i}$ is isomorphic outside $\psi_{i}^{-1}\left(S_{0} \cap U_{i}\right)$. And then we show that these $W_{i}$ and $f_{i}$ are patched together and form the desired variety $X_{1}$ and the morphism $f_{1}$.

First, put $W_{i}=U_{i}, V_{i}=\left\{U_{i}\right\}$, and $\psi_{i}=i d_{\mathfrak{n}_{i}}$ if $U_{i} \cap S_{0}=\phi$.

Suppose then $U_{i} \in \mathfrak{V}$ is such that $U_{i} \cap S_{0} \neq \phi$, then, since $F_{i}=$ $\pi_{i}^{-1}\left(S_{0}\right)$ is coordinate subspace in $C^{m}\left(u^{i}\right)$ of codimension $l$, we may assume that $F_{i}$ is defined by the equations $u_{1}^{i}=\cdots=u_{l}^{i}=0$ in $\mathbb{C}^{m}\left(u^{i}\right)$. Then $g_{i}$ must be of the form $g_{i}=\left(e_{n_{i}}^{p_{1}^{(i)}}, \ldots, e_{n_{i}}^{p_{m}^{(i)}}\right)$ with $p_{k}^{(i)} \neq 0$ for $1 \leqq k \leqq l$, but then by the maximality of $l$, we have $p_{k}^{(i)}=0$ for $k>l$, namely, $g_{i}=\left(e_{n_{i}}^{p_{1}^{(i)}}, \ldots, e_{n_{i}}^{p_{i}^{(i)}}, 1, \ldots, 1\right)$.

Now suppose further that $U_{j}$ is adjacent to $U_{i}$ and $U_{j} \cap S \neq \phi$. Then if $\pi_{i}^{-1}\left(U_{i} \cap U_{j}\right)$ is defined by $u_{k}^{i} \neq 0$ in $\mathbb{C}^{m}\left(u^{i}\right)$, then we must have $k>l$, for otherwise $F_{i}$ would be contained in the hyperplane $u_{k}^{i}=0$ and hence $S_{0} \cap U_{i} \cap U_{j}=\phi$, which contradicts the assumption. Thus we may assume that $k=m$ renumbering the coordinate if necessary. Considering analogously with $U_{j}$, we may also assume that $F_{j}=\pi_{j}^{-1}\left(S_{0}\right)$ (resp. $\pi_{j}^{-1}\left(U_{i}\right.$ $\left.\cap U_{j}\right)$ ) is defined by $u_{1}^{j}=\cdots=u_{l}^{j}=0$ (resp. $u_{m}^{j} \neq 0$ ) in $C_{m}\left(u^{j}\right)$ and $g_{j}=$ $\left(e_{n_{j}}^{p_{1}^{(j)}}, \ldots, e_{n_{j}}^{p_{l}^{(j)}}, 1, \ldots, 1\right)$ on $\boldsymbol{C}^{m}\left(u^{j}\right)$. Then we get the multivalued map $\pi_{i j}$ in he following form;

$$
\begin{aligned}
& u_{k}^{i}=u_{k}^{j}\left(u_{m}^{j}\right)^{a_{k}} \quad \text { if } k \neq i \\
& u_{m}^{i}=\left(u_{m}^{j}\right)^{-n_{j} / n_{i}}
\end{aligned}
$$


Assertion 1. i) For the given generator $g_{i}$ of $G_{i}$, we can tahe $a$ generator $g_{j}$ of $G_{j}$ so that $n_{i}=n_{j}$ and $p_{\alpha}^{(i)}=p_{\alpha}^{(j)}$ for all $\alpha$.

ii) The multivalued map $\pi_{i j}$ above has in fact the following form;

$$
\left\{\begin{array}{l}
u_{k}^{i}=u_{k}^{j}\left(u_{m}^{j}\right)^{a_{k}} \quad \text { if } k \neq i \text { and } k \leqq l \\
u_{k}^{i}=u_{k}^{i}\left(u_{m}^{j}\right)^{a_{k}}, \quad \text { with } a_{k} \text { integers if } k>l \\
u_{m}^{i}=\left(u_{m}^{j}\right)^{-1} .
\end{array}\right.
$$

Proof. Let $G_{i}$ act on $C^{l}\left(u_{1}^{i}, \ldots, u_{l}^{i}\right)$ by $g=\left(e_{n_{l}}^{p_{i}^{(i)}}, \ldots, e_{n_{i}}^{p_{i}^{(i)}}\right)$. Then corresponding to the decomposition $\boldsymbol{C}^{m}\left(u^{i}\right)=\boldsymbol{C}^{l}\left(u_{1}^{i}, \ldots, u_{l}^{i}\right) \times \mathbb{C}^{m-l}\left(u_{l+1}^{i}, \ldots, u_{m}^{i}\right)$, we have an isomorphism $\mathbb{C}^{m} / G_{i} \cong \boldsymbol{C}^{l} / G_{i} \times \boldsymbol{C}^{m-l}$ such that the quotient map $\rho_{i}: \mathbb{C}^{m} \rightarrow \boldsymbol{C}^{m} / G_{i}$ corresponds to the product of $p_{i}^{\prime}: \mathbb{C}^{l} \rightarrow \mathbb{C}^{l} / G_{i}$ and the identity map of $\boldsymbol{C}^{m-l}$. Identify $\mathbb{C}^{m} / G_{i}$ with $\mathbb{C}^{l} / G_{i} \times \mathbb{C}^{m-l}$ by this isomorphism. Then we have the commutative diagram

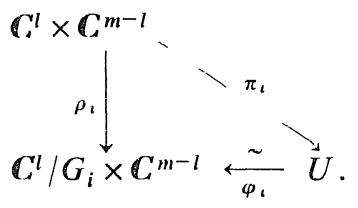

Proceeding analogously with $U_{j}$, we have the similar diagram for $j$. Thus we get $\rho_{i}^{-1} \cdot \varphi_{i}^{-1} \cdot \varphi_{j} \cdot \rho_{j}=\pi_{i}^{-1} \cdot \pi_{j}$ on $\pi_{j}^{-1}\left(U_{i} \cap U_{j}\right)$. From this, we obtain the following commutative diagram

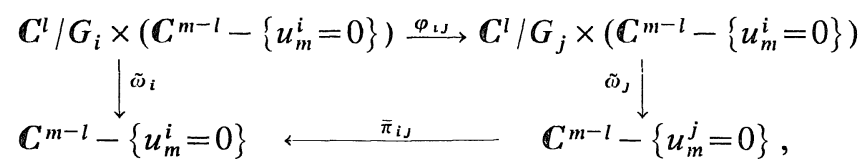

where $\varphi_{i j}=\varphi_{i} \varphi_{j}^{-1} \mid \varphi_{j\left(U_{i} \cap U_{J}\right)}^{-1}, \tilde{\omega}_{i}$ and $\tilde{\omega}_{j}$ are the projections to the second factors and $\bar{\pi}_{i j}$ is the multivalued map defined by $u_{k}^{i}=u_{k}^{j}\left(u_{m}^{j}\right)^{a_{k}}, l+1 \leqq$ $k \leqq m-1$, and $u_{m}^{i}=\left(u_{n}^{j}\right)^{-n_{j} / n_{i}}$. But since $\varphi_{i j}$ is an isomorphism, so must be $\bar{\pi}_{i j}$. In particular it is single-valued. Hence, we conclude that $n_{i}=$ $n_{j}$ and $a_{k}, l+1 \leqq k \leqq m-1$, are all integers. This proves ii). On the other hand, the linear isomorphism $\tilde{\varphi}_{i j}(P): \mathbb{C}^{l} \rightarrow \mathbb{C}^{l}$ defined by $u_{k}^{i}=$ $u_{k}^{j}\left(\bar{u}_{n}^{j}\right)^{a_{k}}, l \geqq k \geqq 1$, for each fixed point $P=\left(\bar{u}_{l+1}^{j}, \ldots, \bar{u}_{m}^{j}\right) \in \mathbb{C}^{m-l}-\left\{u_{m}^{j}=0\right\}$, induces the isomorphism $\varphi_{i j} \mid \tilde{\omega}_{j}^{-1}(P): \boldsymbol{C}^{l} / G_{j}=\mathbb{C}^{l} / G_{i}$. Hence, by $\tilde{\varphi}_{i j}(P)^{-1}$, 
$G_{i}$ must be mapped isomorphically onto $G_{j}$ by Lemma 2 , since both $G_{i}$ and $G_{j}$ are small. But $g_{i}$ is mapped to $g_{j}^{\prime}=\left(e_{n_{i}}^{p_{1}^{(i)}}, \ldots, e_{n_{i}}^{p_{i}^{(i)}}\right)$ by the definition of $\tilde{\varphi}_{i j}(p)$, so we may take this to be the generator of $G_{j}$.

Now fix once and for all a generator $g_{i_{0}} \in G_{i_{0}}$. Then by the admissibility of $(X, \mathfrak{u})$ and by the above assertion, we can successively define a distinguished generator $g_{i}^{\prime}$ of $G_{i}$ for every $i$ for which $U_{i} \cap S_{0} \neq \phi$, with the following properties; if $U_{i}$ is adjacent to $U_{j}$, and is related to $U_{j}$ by the multivalued map $\pi_{i j}$ as above after a suitable renumbering of coordinates depending on $i$ and $j$, then $n_{i}=n_{j}\left(=n_{i_{0}}\right)$ and $p_{k}^{(i)}=p_{k}^{(j)}$, $1 \leqq k \leqq l$. We leave the precise argument to the reader. But for the proof it makes no difference if we change the generator $g_{i}$ to $g_{i}^{\prime}$. So we assume that this change has already been done, and we denote the new generators also by the same letters $g_{i}$. Then by Lemma 43 ) and 5), we see that $\psi_{i}$ is isomorphic outside $\psi_{i}^{-1}\left(S_{0} \cap U_{i}\right)$.

We define now for each $U_{i}, U_{i} \cap S_{0} \neq \phi, W_{i}, \mathfrak{B}_{i}=\left\{V_{1}^{\prime}, \ldots, V_{l}^{i}\right\}$ and $\psi_{i}: W_{i} \rightarrow U_{i}$ as those constructed in Lemma 3, taking $U_{i}=X, G_{i}=G$ and so on.

The next step is to show

Assertion 2. The birational map $\psi_{i j}=\psi_{i}^{-1} \psi_{j}$ gives an isomorphism of $\psi_{j}^{-1}\left(U_{i} \cap U_{j}\right)$ and $\psi_{i}^{-1}\left(U_{i} \cap U_{j}\right)$.

Proof. If either $U_{i} \cap S_{0}=\phi$ or $U_{j} \cap S=\phi$, then this is obvious, because then both $\psi_{i}^{-1}$ and $\psi_{j}^{-1}$ are isomorphic on $U_{i} \cap U_{j}$. So we assume that $U_{i} \cap U_{j} \cap S_{0} \neq \phi$ (note that this is equivalent to $U_{i} \cap S_{0} \neq \phi$ and $U_{i} \cap$ $\left.S_{0} \neq \phi\right)$. If we show that $\psi_{i j}$ is holomorphic at each point of $\psi_{j}^{-1}\left(U_{i} \cap\right.$ $U_{j}$ ) as a map of analytic spaces, then by Z.M.T., we see that this is a morphism of varieties, and since this holds also for $\psi_{i j}$, we get that $\psi_{i j}$ is an isomorphism. To prove that $\psi_{i j}$ is holomorphic, we go back to the situation and the notations given in the proof of the Assertion 1. There, we defined for each point $P=\left(\bar{u}_{l+1}^{j}, \ldots, \bar{u}_{m}^{j}\right) \in C^{m-l}-\left\{u_{m}^{j}=0\right\}$, the linear map $\tilde{\varphi}_{i j}(P): \boldsymbol{C}^{l} \rightarrow \boldsymbol{C}^{l}$. But if we take a simply connected subdomain $D$ in $C^{m-l}-\left\{u_{m}^{j}=0\right\}$ and choose a suitable branch for each $\left(\bar{u}_{m}^{j}\right)^{a_{k}}$, the same formula with $\tilde{\varphi}_{i j}(P)$ defines the isomorphism $\tilde{\varphi}_{i j}(D)$ : 
$C^{l} \rightarrow C^{l}$ depending holomorphically on $D:$ Further this fits into the following commutative diagram;

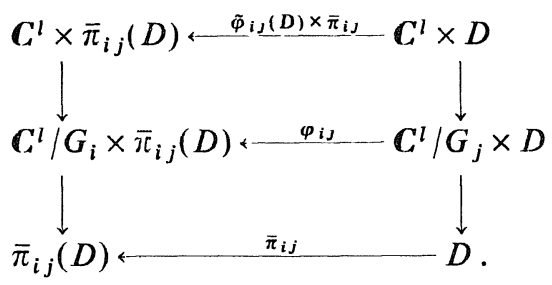

From this we infer readily that $\psi_{i j}$ is holomorphic on $\psi_{j}^{-1}(D)$ by the definition of $\psi_{j}$.

Q.E.D.

Now we define $X_{1}$ to be the union $X_{1}=\bigcup_{i=1}^{s} W_{i}$, where if $U_{i}$ is adjacent to $U_{j}$, then $\left(w^{i}\right) \in W_{i}^{\prime}$ and $\left(w^{j}\right) \in W_{j}$ are identified if and only if $\left(w^{i}\right) \in \psi^{-1}\left(U_{i} \cap U_{j}\right) \quad\left(\operatorname{resp} .\left(w^{j}\right) \in \psi_{j}^{-1}\left(U_{i} \cap U_{j}\right)\right)$ and $\psi_{j i}\left(w^{i}\right)=\left(w^{j}\right)$. Then define $f_{1}: X_{1} \rightarrow X$ by the condition that $f_{1} \mid W_{i}=\psi_{i}$. It is easy to see that by the definition, $X_{1}$ becomes a variety and $f_{1}$ a morphism of varieties.

Moreover we shall prove

Assertion 3. The pair $\left(X_{1}, \mathfrak{l}_{1}\right)$ is admissible, where $\mathfrak{U}_{1}$ is the union of the ocverings $\mathfrak{B}_{i}$.

Proof. First we fix $U_{i}$ and $U_{j}$ which are mutually adjacent and make some preliminary considerations. We distinguish three cases; 1) neither $U_{i}$ nor $U_{j}$ intersects with $\left.S_{0}, 2\right)$ either $U_{i}$ or $U_{j}$ intersects with $S_{0}$ but not both, and 3) both $U_{i}$ and $U_{j}$ intersects with $S_{0}$.

In case 1 ), since $W_{i}$ (resp. $\left.W_{j}\right)=U_{i}$ (resp. $U_{j}$ ) by construction, $W_{i}$ is adjacent to $W_{j}$ and the corresponding multivalued map satisfies the condition $(\beta)$.

Next we consider the case 3 ). We may assume that $\pi_{i j}$ is given by (4). Then $W_{i}$ (resp. $W_{j}$ ) is covered by $l$ affine open subsets $V_{i 1}, \ldots, V_{i l}$ (resp. $\left.V_{j 1}, \ldots, V_{j l}\right)$ and the multivalued map $\pi_{i}^{-1} \psi_{i} \pi_{i s}: \boldsymbol{C}^{m}\left(v^{i s}\right) \rightarrow \boldsymbol{C}^{m}\left(u^{i}\right)$, $1 \leqq s \leqq l$, has the form; 


$$
\begin{cases}u_{s}^{i}=\left(V_{s}^{i s}\right)^{n_{\imath s} / n_{i}} & \\ u_{k}^{i}=\left(V_{s}^{i s}\right)^{b_{k}} v_{k}^{i s} & \text { for } \quad 1 \leqq k \leqq l \text { and } k \neq s \\ u_{k}^{i}=v_{k}^{i s} & \text { for } \quad m \geqq k \geqq l+1,\end{cases}
$$

where $\pi_{i s}: \mathbb{C}^{m}\left(v^{i s}\right) \rightarrow V_{i s}$ is the quotient map, and $b_{k}$ are certain rational numbers. Here we write down also the inverse to the above map for convenience.

$$
\begin{cases}v_{s}^{i s}=\left(u_{s}^{i}\right)^{n_{i} / n_{i s}} & \\ v_{k}^{i s}=u_{k}^{i}\left(u_{s}^{i}\right)^{-n_{i} b_{k} / n_{i s}} & \text { for } 1 \leqq k \leqq l \text { and } k \neq s \\ v_{k}^{i s}=u_{k}^{i} & \text { for } m \geqq k \geqq l+1 .\end{cases}
$$

These follow from Lemma 4. From this, it is easy to see that $V_{i s}$ and $V_{j s}$ are adjacent to each other, and no other two $V_{i k}$ and $V_{j k^{\prime}}$ are adjacent. Moreover since $\pi_{i j}=\pi_{i}^{-1} \pi_{j}=\left(\pi_{i} \psi_{i} \pi_{i k}\right)^{-1}\left(\pi_{i}^{-1} \pi_{j}\right)\left(\pi_{j}^{-1} \psi_{j} \pi_{j s}\right)$, using (4) and (5) we infer that this has the following form;

$$
\left\{\begin{array}{l}
v_{s}^{i s}=\left(v_{m}^{j s}\right)^{\frac{n_{i} a_{s}}{n_{i s}}} v_{s}^{j s} \\
v_{k}^{i s}=\left(v_{m}^{j s}\right)^{\frac{n_{i s} a_{k}-a_{s} n_{i} b_{k}}{n_{i s}}} v_{k}^{j s}, k \neq s \\
v_{m}^{i s}=\left(v_{m}^{j s}\right)^{-1}
\end{array}\right.
$$

Hence the condition $(\beta)$ is satisfied for $V_{i s}$ and $V_{j s}$.

Finally, we deal with the case 2), say, when $U_{j} \cap S_{0} \neq \phi$. The alternative case can be treated analogously. Then if $\pi_{i}^{-1}\left(U_{i} \cap U_{j}\right)$ is defined by $u_{s}^{i} \neq 0$, then $1 \leqq s \leqq l$. For, otherwise, $U_{i} \cap S_{0} \neq \phi$. Hence we may assume that the multivalued map $\pi_{i j}$ has the form $u_{k}^{i}=\left(u_{s}^{j}\right)^{a_{k}} u_{k}^{j}$ for $k \neq s$ and $u_{s}^{i}=\left(u_{s}^{i}\right)^{n_{j} / n_{i}}$. Then we see readily that $V_{i s}$ is the only element which is adjacent to $W_{j}$ and that $\pi_{i}^{-1} \pi_{j s}=\left(\pi_{i}^{-1} \pi_{j}\right)\left(\pi_{j}^{-1} \psi_{j} \pi_{j s}\right)$ takes the following form;

$$
\left\{\begin{array}{l}
u_{k}^{i}=\left(v_{s}^{j_{s}}\right)^{\frac{n_{j_{s} a_{n}+b_{k} n_{j}}}{n_{j}}} v_{k}^{j s}, k \neq s \\
u_{s}^{i}=\left(v_{s}^{j s}\right)^{-n_{j s} / n_{i}}
\end{array}\right.
$$


This gives the condition $(\beta)$ for $W_{i}$ and $V_{j s}$.

Using these results we shall now prove the condition $(\alpha)$ for $\left(X_{1}\right.$, $\left.\mathfrak{U}_{1}\right)$. So suppose $V_{i}^{\alpha}$ is adjacent to $V_{j}^{-\beta}$. Then, by the admissibility of $(X, \mathfrak{U})$, there exists a finite sequence $U_{0}, \ldots, U_{t}$ such that $U_{0}=U_{i}, U_{t}=$ $U_{j}$, and $U_{\mu}$ is adjacent to $U_{\mu+1}$ for $0 \leqq \mu \leqq t-1$. Then, by the previous considerations, there exist for each successive pair $\left(U_{k}, U_{k+1}\right), 0 \leqq k<t$, $V_{\alpha(k)} \in \mathfrak{B}_{k}$ and $V_{\beta(k)} \in \mathfrak{V}_{k+1}$ such that $V_{\alpha(k)}$ is adjacent to $V_{\beta(k)}$. But by virtue of Lemma $4 V_{\alpha(k+1)}$ is adjacent to $V_{\beta(k)}$. Hence, $V_{\alpha}, V_{\alpha(0)}, V_{\beta(0)}, \ldots$, $V_{\beta(t-1)}, V_{\beta}$ is the sequence of adjacent members of $\mathfrak{U}_{1}$, which connects $V_{\alpha}$ and $V_{\beta}$. This proves $(\alpha)$.

Next, in order to see the condition $(\beta)$, again by the previous consideration, we have only to show that if $V_{\alpha}$ is adjacent to $V_{\beta}$ and if $V_{\alpha} \in \mathfrak{B}_{i}$ and $V_{\beta} \in \mathfrak{B}_{j}$, then $U_{i}$ is adjacent to $U_{j}$. But this is easy to check by virtue of Lemma 4 , iii).

Finally, the groups $G_{i}$ are small by construction. This is $(\gamma)$, thus completing the proof of the assertion.

In order to apply the induction hypothesis to the admissible pair $\left(X_{1}, \mathfrak{U}_{1}\right)$, we have to see that $\varphi_{X_{1}}<\varphi_{X}$. But by construction we see that for any $V_{\alpha} \in \mathfrak{B}_{i}$ if $U_{i} \cap S_{0} \neq \phi$, then ord $V_{\alpha}<\operatorname{ord} U_{i}$ and if $U_{i} \cap S_{0}=\phi$, ord $V_{\alpha}=\operatorname{ord} U_{i}$, where ord $V_{\alpha}$ (resp. ord $U_{i}$ ) denotes the order of $V_{\alpha}$ (resp. of $U_{i}$ ). Hence $\varphi_{X_{1}}<\varphi_{X}$.

Hence by the induction hypothesis there exist a resolution $\left(\tilde{X}, f_{2}\right)$ of $X_{1}$ and a finite affine open covering $\tilde{U}$ of $\tilde{X}$ satisfying the properties stated in the theorem. Hence 2) $f_{2}$ is admissible as a morphism of admissible pairs $(\tilde{X}, \tilde{\mathfrak{U}})$ and $\left.\left(X_{1}, \mathfrak{U}_{1}\right), 3\right)_{1}$ any admissible automorphism of $\left(X_{1}, \mathfrak{U}_{1}\right)$ extends uniquely onto that of $(\tilde{X}, \tilde{\mathfrak{U}})$, and 4$)_{1} f_{2}^{-1}\left(S_{1}\right) \cap \tilde{U}_{s}$ is defined by $\tilde{u}_{k_{1}}^{s} \ldots \tilde{u}_{k_{\mu}}^{s}=0$ for some $k_{1}, \ldots, k_{\mu}$ if it is not empty, where $S_{1}$ is the singular locus of $X_{1}$. Set $f=f_{2} \cdot f_{1}$, then since $f_{1}$ is isomorphic outside $f_{1}^{-1}\left(S_{0}\right)$ and $S_{0} \subseteq S$, we see that $(\tilde{X}, f)$ gives a resolution of $X$. We show that these $(X, f)$ and $(\tilde{X}, \tilde{\mathfrak{u}})$ satisfy the requirement of the theorem. For this, we have to check the conditions 2), 3) and 4) of the theorem. Note first that $f_{1}$ is admissible since so is each $\psi_{i}$ by Lemma 4,3$)$. Then it is clear that $f$ is admissible as a composition of two admissible morphisms $f_{1}$ and $f_{2}$. This checks 2). Next, let $T$ be an admissible automorphism of $U_{i}$, then by Lemma 4 this extends 
uniquely onto an admissible automorphism of $\left(W_{i}, \mathfrak{B}_{i}\right)$ and this in turn has the unique extension to an admissible automorphism $f^{-1}\left(\left(U_{i},\left\{U_{i}\right\}\right)\right)$ by 3$)_{1}$. This is 3). Finally we deal with 4). Note first that $f^{-1}(S)=$ $f_{2}^{-1}\left(S_{1}\right) \cup f_{2}^{-1}\left(f_{1}^{-1}\left[S_{0}\right]\right)$, where $f_{1}^{-1}\left[S_{0}\right]$ is the proper transform of $S_{0}$ in $X_{1}$. By virtue of 4$)_{1}$ above it is enough to see that $f_{2}^{-1}\left(f_{1}^{-1}\left[S_{0}\right]\right) \cap$ $\tilde{U}_{s}$ is defined by the equation $\tilde{u}_{k_{1}}^{s} \tilde{u}_{k_{2}}^{s} \ldots \tilde{u}_{k_{\mu}}^{s}=0$ on $\tilde{U}_{s}$ if it is not empty. But this follows from 5) of Lemma 4 and the admissibility of $f$. This completes the proof of the theorem.

Remark 3. a) From our construction the resolution map $f$ is seen to be naturally decomposed into $f=f_{1} \ldots f_{d}$, such that $f_{i}: X_{i} \rightarrow X_{i-1}, 1 \leqq i \leqq$ $d$, are proper birational morphisms with $X_{0}=X$ and $X_{d}=\tilde{X}$ whose exceptional loci $E_{i}$ are irreducible. Moreover if we put $s(i)=\operatorname{dim} f_{i}\left(E_{i}\right)$, then the function $s(i)$ is nondecreasing for $1 \leqq i \leqq d$. Further if $s(i)$ is constant for $a \leqq i \leqq b$ and if we put $f_{1} \ldots f_{i}\left(E_{i}\right)=E_{a}^{i}$, then these $E_{a}^{i}, a \leqq i \leqq b$, are disjoint. Hence we have $f_{i} f_{i+1}=f_{i+1} f_{i}$ for $a \leqq i, i+1 \leqq b$ in an obvinus sense. For example let $X=N_{n, p_{1}, \ldots, p_{m}}$ be a cyclic quotient singularity. Then $s(i)=0$ for all $i$ if and only if $n, p_{1}, \ldots, p_{m}$ are all relatively prime as follows from Lemma 4 and Remark $1 b$ ).

b) In constructing a resolution of an admissible pair $(X, \mathfrak{U})$ according to the inductive method described in the proof of the theorem, we have a finite number of choices in taking generators of $G_{i}$, or taking isomorphism $\varphi_{i}: U_{i} \cong N_{n_{i}, p_{1}^{(i)}, \ldots, p_{m}^{(i)}}$ at each step. In general different choices of generators lead to different resolutions, as was indicated in the remark at the end of 1.2 (see an example in 1.4).

c) We say a resolution $f: \tilde{X} \rightarrow X$ is special if in each step $f_{i}: X_{i} \rightarrow$ $X_{i-1}$ of the resolution, the isomorphisms $\varphi_{i}: U_{i} \cong N_{n_{i}, p_{1}^{(i)}, \ldots, p_{m}^{(i)}}$ are taken so that $p_{k}^{(i)}=1$ for some $k$. If $X=N_{n, p_{1}, \ldots, p_{q}}$ and $\left(p_{i}, n\right)=1$ for some $i$, then a special resolution of $X$ exists as follows from Lemma 3. For the motivation for this definition, we refer to Remark 6 after Proposition 1 in the next section.

1.4. By way of illustration we apply the above method when $m=2$, and next, examine the case when $m=3$.

1.4.1. Let $X=C^{2} / G$ be the cyclic quotient singularity of dimension 2 . 
If the group $G$ is small, then it is easy to see that there exists a unique generator $g \in G$ such that $g=\left(e_{n}, e_{n}^{p}\right)$ with $0 \leqq p<n$ and $(p, n)=1$ with respect to the coordinate $\left(z_{1}, z_{2}\right)$ of $\boldsymbol{C}^{2}$. Then taking $g$ as a generator of $G$ we construct $X_{1}, \mathfrak{H}=\left\{U_{1}, U_{2}\right\}$ and $f: X_{1} \rightarrow X$ as in 1.2. Then by Lemma $3 U_{1} \cong \boldsymbol{C}^{2}\left(u_{1}, v_{1}\right)$ and $U_{2} \cong N_{p, 1, p_{1}}$, where $p_{1}$ is defined by the following formula; $0 \leqq p_{1}<p, \quad n=b_{1} p-p_{1}, \quad b_{1} \geqq 2$. Next applying the same process to $U_{2}=N_{p, 1, p_{1}}$, we have a variety $X_{2}$.with an affine open covering $\left\{U_{1}, U_{21}, U_{22}\right\}$ such that $U_{21} \cong \boldsymbol{C}^{2}\left(u_{2}, v_{2}\right)$ and $U_{22} \cong N_{p_{1}, 1, p_{2}}$, where this time $p_{2}$ is defined by $0 \leqq p_{2}<p_{1}, p=b_{2} p_{1}-p_{2}, b_{2} \geqq 2$. Then using 2) and 3) of Lemma 4 the transition functions between $U_{1}$ and $U_{21}$ are calculated as follows;

$$
\left\{\begin{array}{l}
u_{1}=\left(u_{2}\right)^{b_{1}} v_{2} \\
v_{1}=\left(u_{2}\right)^{-1}
\end{array}\right.
$$

Continueing analogously we are finally led to the minimal resolution $f$ : $\tilde{X} \rightarrow X$ of $X$ first constructed by Hirzebruch in [3] (see also [12]): Define positive integers $\lambda_{k}, \mu_{k} 0 \leqq k<s+1$, and $b_{k}, 1 \leqq k \leqq s$ by the formula;

$$
\left\{\begin{array}{l}
\lambda_{0}=n \quad \lambda_{1}=p \quad \lambda_{k+1}=b_{k} \lambda_{k}-\lambda_{k-1}, \quad b_{k} \geqq 2,0 \leqq \lambda_{k-1}<\lambda_{k} \lambda_{s}=1 \lambda_{s+1}=0 \\
\mu_{0}=0 \quad \mu_{1}=1 \quad \mu_{k+1}=b_{k} \mu_{k}-\mu_{k-1}
\end{array}\right.
$$

Then $\tilde{X}$ is covered by $s+1$ copies $W_{h}, 0 \leqq k \leqq s$, of complex affine place $C^{2}$ with the coordinate $\left(u^{k}, v^{k}\right)$ and the transition functions between $W_{k-1}$ and $W_{k}$ are given by the following formula;

$$
\left\{\begin{array}{l}
u_{k}=1 / v_{k-1} \\
v_{k}=\left(v_{k-1}\right)^{b_{k}} u_{k-1} . \quad k=1, \ldots, s .
\end{array}\right.
$$

The rational map $T: C^{2}\left(z_{1}, z_{2}\right) \rightarrow \tilde{X}$ is given by

$$
T_{k}:\left(z_{1}, z_{2}\right) \longrightarrow\left(z_{1}^{\lambda_{k}} z_{2}^{-\mu_{k}}, z_{1}^{-\lambda_{k+1}} z_{2}^{\mu_{k+1}}\right) \in W_{k} .
$$

Further if we define nonsingular subvarieties $\theta_{k}, 1 \leqq k \leqq s$, on $\tilde{X}$ by

$$
\left\{\begin{array}{l}
u_{s-k}=0 \quad \text { on } \quad W_{s-k} \\
v_{s-k+1}=0 \quad \text { on } \quad W_{s-k+1}
\end{array}\right.
$$


then $f$ is isomorphic outside $\theta=\bigcup_{i=1}^{k} \theta_{i}, \theta_{k} \cong \mathbb{P}^{1}$ and the selfintersection number $\theta_{k} \cdot \theta_{k}=-b_{k}$. Hence in particular there are no exceptional curves of the first kind in $f^{-1}(\mathfrak{W})$, where $\mathfrak{P}$ is the singular point of $X$. But if we make no normalization of the generator as above, then the resolution is in general not minimal. For instance, let $X=C^{2} / G$ and $G=\{g\}$ with $g$ acting on $\mathbb{C}^{2}$ by $g=\left(e_{5}^{3}, e_{5}^{4}\right)$. Further let $f: \tilde{X} \rightarrow X$ be the resolution of $X$ obtained in the theorem and let $\mathfrak{P} \in X$ the singular point of $X$. Then $f^{-1}(\mathfrak{P})$ consists of nonsingular rational curves $C_{1}, C_{2}$, and $C_{3}$ with intersection numbers $\left(C_{1}\right)^{2}=-3,\left(C_{2}\right)^{2}=-1,\left(C_{2}\right)^{2}=-4, C_{1} \cdot C_{2}$ $=1, C_{2} \cdot C_{3}=1$ and $C_{1} \cdot C_{3}=0$.

1.4.2. So we may consider some kind of normalizations also in the higher dimensional cases, and expect a certain minimality condition for the resolutions. But in the following we shall restrict ourselves half for simplicity to the case when $m=\operatorname{dim} X=3$, and assume that $\left(n, p_{1}\right)=1$. In this case we can take the canonical generator $g \in G$ by the condition that $g=\left(e_{n}, e_{n}^{p}, e_{n}^{q}\right)$, where $0 \leqq p, q<n$ and $(n, p, q)=1$. Here we have assumed that the group $G$ is small (c.f. Remark 1). Then we often write $X=N_{n ; p . k}$ instead of $X=N_{n, 1, p, q}$.

Now suppose $X_{1}, \mathfrak{H}_{1}=\left\{U_{1}, U_{2}, U_{3}\right\}$ and $f_{1}: X_{1} \rightarrow X$ are as in Lemma 3. Then since $q_{2}^{\frac{1}{2}}=q_{3}^{1}=1$, we have the canonical isomorphisms

$$
U_{1} \cong \mathbb{C}^{3}, U_{2} \cong N_{p ; p_{2} p_{3}} \text { and } \quad U_{3} \cong N_{q: q_{2} q_{3}},
$$

where $p_{2}, p_{3}, q_{2}$ and $q_{3}$ are determined by the following formulas;

$$
\begin{aligned}
& p_{2}+n \equiv 0 \bmod p, \quad p_{3} \equiv q \bmod p \text { and } 0 \leqq p_{i}<p \\
& q_{2} \equiv p \bmod q \quad q_{3}+n \equiv 0 \bmod q \text { and } 0 \leqq q_{i}<q .
\end{aligned}
$$

By Lemma 4 the transition functions $\pi_{i j}$ and the rational map $X_{1} \rightarrow$ $\mathbb{C}^{3}(z)$ are given respectively by

$$
\left\{\begin{array} { l } 
{ w _ { 1 } ^ { 2 } = ( w _ { 2 } ^ { 1 } ) ^ { - 1 / p } } \\
{ w _ { 2 } ^ { 2 } = w _ { 1 } ^ { 1 } ( w _ { 2 } ^ { 1 } ) ^ { n / p } } \\
{ w _ { 3 } ^ { 2 } = w _ { 3 } ^ { 1 } ( w _ { 2 } ^ { 1 } ) ^ { - q / p } }
\end{array} \quad \left\{\begin{array} { l } 
{ w _ { 1 } ^ { 3 } = ( w _ { 3 } ^ { 1 } ) ^ { - 1 / q } } \\
{ w _ { 2 } ^ { 3 } = w _ { 2 } ^ { 1 } ( w _ { 3 } ^ { 1 } ) ^ { - p / q } } \\
{ w _ { 3 } ^ { 3 } = w _ { 1 } ^ { 1 } ( w _ { 3 } ^ { 1 } ) ^ { n / q } }
\end{array} \left\{\begin{array}{l}
w_{1}^{2}=w_{1}^{3}\left(w_{2}^{3}\right)^{-1 / p} \\
w_{2}^{2}=w_{3}^{3}\left(w_{2}^{3}\right)^{n / p} \\
w_{3}^{2}=\left(w_{2}^{3}\right)^{-q / p}
\end{array}\right.\right.\right.
$$




$$
\left\{\begin{array}{l|l|l}
z_{1}=\left(w_{1}^{1}\right)^{1 / n} & z_{1}=w_{1}^{2}\left(w_{2}^{2}\right)^{1 / n} \\
z_{2}=w_{2}^{1}\left(w_{1}^{1}\right)^{p / n} & z_{2}=\left(w_{2}^{2}\right)^{p / n} & z_{1}=w_{1}^{3}\left(w_{3}^{3}\right)^{1 / n} \\
z_{3}=w_{3}^{1}\left(w_{1}^{1}\right)^{q / n} & z_{3}=w_{1}^{3}\left(w_{2}^{2}\right)^{q / n} & z_{2}=w_{2}^{3}\left(w_{3}^{3}\right)^{p / n} \\
z_{3}=\left(w_{3}^{3}\right)^{q / n}
\end{array}\right.
$$

From this we see that we can define the canonical way of resolution for such $X$. To state this precisely, we shall consider some preliminary cases. So suppose $X=N_{n, 1, p} \times C$ and $h_{0}: \tilde{N}_{n, 1, p} \rightarrow N_{n, 1, p}$ is the minimal resolution. Let $h=h_{0} \times i d_{\boldsymbol{C}}: \tilde{N}_{n, 1, p} \times \boldsymbol{C} \rightarrow N_{n, 1, p} \times \boldsymbol{C}$ be the resolution of $X$ obtained as the product of $h_{0}$ and the identity of $C$. Then we say that the resolution $h$ of $X$ is minimal. Next suppose $(X, \mathfrak{U})$ with $\mathfrak{U}=$ $\left\{U_{1}, U_{2}\right\}$ is an admissible pair. Assume that $U_{i}=C^{3}\left(u^{i}, v^{i}, w^{i}\right) /\left\{g_{i}\right\}$ and $g_{i}=\left(e_{n}, e_{n}^{p}, 1\right), i=1,2$. Obviously each $U_{i}$ is isomorphic to $N_{n, 1, p} \times \mathbb{C}$. Then the minimal resolutions of $U_{i}$ coincides on the intersection $U_{1} \cap U_{2}$ and gives a resolution $f: \tilde{X} \rightarrow X$ of $X$. We call this the minimal resolution of $X$. Further in this case if the transition functions between $U_{1}$ and $U_{2}$ are given by the formula

$$
\left\{\begin{array}{l}
u^{i}=u^{i}\left(w^{j}\right)^{a_{1}} \\
\iota^{i}=v^{j}\left(w^{j}\right)^{a_{2}} \\
w^{i}=\left(w^{j}\right)^{-1}
\end{array}\right.
$$

with $a_{i}$ some rational numbers, then $X$ is covered by $2 s$ copies $v_{k}^{i}, 1 \leqq$ $k \leqq s, i=1,2$, of $\boldsymbol{C}^{3}$ with the coordinates $\left(u_{h}^{i}, v_{h}^{i}, w_{k}^{i}\right)$ such that the transition functions between them are given by the following formula;

$$
\begin{cases}u_{k}^{i}=\left(v_{k-1}^{i}\right)^{-1} & u_{k}^{i}=u_{k}^{2}\left(w_{k}^{2}\right)^{a_{1} \lambda_{k}-a_{2} \mu_{k}} \\ v_{k}^{i}=\left(v_{k-1}^{i}\right)^{b_{h}} u_{k-1}^{i} & v_{k}^{1}=v_{k}^{2}\left(w_{k}^{2}\right)^{-a_{1} \lambda_{k+1}+a_{2} \mu_{k+1}} \\ w_{k}^{i}=w_{k-1}^{i} & w_{k}^{1}=\left(u_{k}^{2}\right)^{-1}\end{cases}
$$

where $b_{k}$ and $\lambda_{k}, \mu_{k}$ are defined by (9). This can be proved easily if one uses (10). Note that the minimal resolution is unique.

Now suppose $X=N_{n ; p, q}$ as before and $f: \tilde{X} \rightarrow X$ is one of the resolutions obtained in the theorem. Decompose $f$ into $f=f_{1} \ldots f_{d}$ as in the 
Remark $3 a$ ). Let $E_{i}$ be the exceptional locus of $f_{i}: X_{i} \rightarrow X_{i-1}$ and set $D_{i}=f_{i}\left(E_{i}\right) . \quad D_{i}$ is either a single point or isomorphic to a projective line. We may assume that $D_{i}$ is a point for $i \leqq b$ and is a line if $i>b$ (c.f. Remark $3 a$ )). Each $X_{i}$ has a natural affine open covering $\mathfrak{U}_{i}$ such that $\left(X_{i}, \mathfrak{H}_{i}\right)$ is an admissible pair. If $D_{i}$ is a point, then there exists a unique member $U_{\alpha}^{(i-1)} \in \mathfrak{H}_{i-1}$ such that $D_{i} \in U_{\alpha}^{(i-1)}$ and $f_{i} \mid f_{i}^{-1}\left(U_{\alpha}^{(i-1)}\right)$ : $f_{i}^{-1}\left(U_{\alpha}^{(i-1)}\right) \rightarrow U_{\alpha}^{(i-1)}$ is the map which replaces $U_{\alpha}^{(i-1)}$ by three affine open sets $U_{\alpha 1}^{(i)}, U_{\alpha 2}^{(i)}$, and $U_{\alpha 3}^{(i)}$ according to the method of 1.2. We say that $f_{1} \ldots f_{i}, 1 \leqq i \leqq b$, is canonically defined if inductively 1) $f_{1} \ldots f_{i-1}$ is canonically defined and 2) $f_{i} \mid f_{i}^{-1}\left(U_{\alpha}^{(i-1)}\right)$ is with respect to the isomorphisms

$$
U_{\alpha 1}^{(i)} \cong C^{3}, U_{\alpha 2}^{(i)}=N_{n_{\alpha 2}^{(1)} ; p_{\alpha 2}^{(t)} q_{\alpha 2}^{(i)}} \text { and } \quad U_{\alpha 3}^{(i)} \cong N_{n_{\alpha 3}^{(1)}, p_{\alpha 3}^{(i)} q_{\alpha 3}^{(i)}}
$$

prescribed in Lemma 3 as explained above for suitable integers $n_{\alpha k}^{(i)}$, $p_{\alpha k}^{(i)}, q_{\alpha k}^{(i)}$. Next, consider the admissible pair $\left(X_{b}, \mathfrak{U}_{b}\right)$. The singular locus of $X_{b}$ is the disjoint union of nonsingular curves $C_{i}, i=1, \ldots, l$, each isomorphic to a projective line. For each $C_{i}$ there exists a unique pair $\left(U_{\alpha}^{(b)}, U_{\beta}^{(b)}\right)$ of the members of $\mathfrak{U}_{b}$ such that $\left(U_{\alpha}^{(b)} \cup U_{\beta}^{(b)},\left\{U_{\alpha}^{(b)}\right.\right.$, $\left.\left.U_{\beta}^{(b)}\right\}\right)$ is an admissible pair of the type considered before. So we may speak of the minimal resolution of $X$ along each $C_{i}$. Then

Definition 3. We say that the resolution $f$ is canonical, or $f$ is the canonical resolution of $X$, if i) $f_{1} \cdots f_{b}$ is canonically defined and ii) $f_{b+1} \ldots f_{d}$ defines the minimal resolution of each $C_{i}$.

The canonical resolution is one of the special resolutions defined in Remark $3 c$ ).

To describe the minimality condition, we make the following definition after Moishezon.

Definition 4. Suppose $\tilde{X}$ is a complex manifold of dimension 3 and $S$ is a connected submanifold of $X$ of codimension 1 . Let $N_{S / X}$ be the normal bundle of $S$ in $X$. We say that $S$ is the exceptional surface of the first kind, if either $S$ is isomorphic to $\boldsymbol{P}^{2}$ and $N_{S / \tilde{X}} \cong-H_{\boldsymbol{P}^{2}}$, or $S$ is isomorphic to a $\boldsymbol{P}^{1}$ bundle over a manifold of dimension 1 and $\left.N_{S / \tilde{X}}\right|_{F} \cong-H_{F}$, where $H$ is the hyperplane bundle of the corresponding projective space, and $F$ is the general fiber of the fibering of $S$. 
Remark 4. If an exceptional surface of the first kind $S$ is compact and rational, then either $S \cong \boldsymbol{P}^{2}$ or $S \cong \Sigma_{m}$, the Hirzebruch surface of degree $m . \quad \Sigma_{m}$ is a $\boldsymbol{P}^{1}$ bundle over $\boldsymbol{P}^{1}$ obtained by adding an $\infty$ section to a line bundle of degree $-m$ on $\boldsymbol{P}^{1}$. We call the 0 -section of this line bundle also the 0 -section of $\Sigma_{m}$. In particular, $\Sigma_{0}=\mathbb{P}^{1} \times$ $\boldsymbol{P}^{1}$ and it has two different fiberings of $\boldsymbol{P}^{1}$ associated to the projections to the first and to the second factors. Conversely, it is known that $\Sigma_{0}$ is the only one among $\Sigma_{m}$ which has two structures of $\boldsymbol{P}^{1}$ bundles.

Now let $f: \tilde{X} \rightarrow X$ be the canonical resolution of $X=N_{n, p, q}$. Let $S$ be the singular locus of $X, \theta=f^{-1}(S)$ and $\theta_{1}, \ldots, \theta_{c}$ be the irreducible components of $\theta$. Let $f=f_{1}, \ldots, f_{d}$ be the decomposition of $f$ as in Remark 3. Suppose some $\theta_{\alpha} \cong \Sigma_{0}$ and is the proper transform of the exceptional locus of $f_{i}: X_{i} \rightarrow X_{i-1}$. Then either of its fiberings $\mu: \theta_{\alpha} \rightarrow \mathbb{P}^{1}$ is said to be incompatible with $f$ if $f_{i}, \ldots, d_{d}\left(\theta_{\alpha}\right)$ is a curve $C_{\alpha}$ and $f_{i}, \ldots$, $f_{d}$ sends each fiber of $\mu$ onto $C_{\alpha}$. Then we can prove

Proposition 1. Suppose $f: \tilde{X} \rightarrow X$ is the canonical resolution of $X=$ $N_{n ; p, q}$ and $S, \theta$ and $\theta_{i}$ are as above. Then none of $\theta_{\alpha}$ are exceptional surfaces of the first kind except when $\theta_{\alpha} \cong \Sigma_{0}$ and $\left.N_{\theta_{\alpha} / X}\right|_{F} \cong-H_{F}$, where $F$ is the general fiber of the fibcring of $\theta_{\alpha}$ incompatible with $f$.

Proof. Let $f=f_{1}, \ldots, f_{d}$ be the decomposition of $f$ as in Remark 3 and $E_{i}$ the exceptional locus of $f_{i}: X_{i} \rightarrow X_{i-1}$. Let $\tilde{E}_{i}$ be the proper transform of $E_{i}$ in $\tilde{X}$. We have to show that $\tilde{E}_{i}$ are not the exceptional surface of the first kind unless it comes under the above exceptional case. Set $D_{i}=f_{i}\left(E_{i}\right)$. First we consider the case when $\operatorname{dim} D_{i}=0$. Then $E_{i}$ is isomorphic to a projective plane divided by a cyclic group. Indeed, by Lemma 3 and 5) of Lemma 4 we see that $E_{i}$ is covered by 3 affine open subsets $V_{1}, V_{2}, V_{3}$, each isomorphic to $C^{2}, N_{p^{\prime}, 1, p_{3}^{\prime}}$, and $N_{q^{\prime}, q_{2}^{\prime}, 1}$ respectively, where we assumed that there exists $U_{\alpha} \in \mathfrak{U}^{(i-1)}$ with the canonical isomorphism with $N_{n^{\prime} ; p^{\prime} q^{\prime}}$ such that $D_{i} \in \mathfrak{U}_{\alpha}$ and where $p_{3}^{\prime}$ and $q_{2}^{\prime}$ are determined from $p^{\prime}$ and $q^{\prime}$ by the formula corresponding to (11). But since $\widetilde{E}_{i}$ are nonsingular by the theorem, the induced map $\widetilde{E}_{i} \rightarrow E_{i}$ gives the resolution of the singularity of $E_{i}$. Now since $\Sigma_{m}$ (resp. $P^{2}$ ) have 2 (resp. 1) as the second betti number, from this, we can 
readily infer that $\tilde{E}_{i}$ is isomorphic to neither of them unless in the following three cases; i) $\left(p_{3}^{\prime}, q_{2}^{\prime}\right)=(0,0)$, ii) $\left(p_{3}^{\prime}, q_{2}^{\prime}\right)=(1,0)$ (iii) $\left(p_{3}^{\prime}, q_{2}^{\prime}\right)=$ $(0,1)$. But i) is equivalent to $p=q$ ii) to $p=1$ and iii) to $q=1$. These cases are dealt with in the following two lemmas.

Lemma 5. (Ueno [12]) Suppose $f: \tilde{X} \rightarrow X$ is the canonical resolution of $X=N_{n ; p, p}$. Then $\tilde{X}$ is covered by $2 s+1$ copies $V_{k}, 1 \leqq k \leqq s$, $i=1,2$, and $V_{0}$, of $C^{3}$ with the coordinates $\left(u_{k}^{i}, v_{k}^{i}, w_{k}^{i}\right)$ and $\left(u^{0}, v^{0}, w^{0}\right)$ respectively and they are connected by the system of transition functions as follows;

$$
\left\{\begin{array} { l } 
{ u _ { k } ^ { i } = ( v _ { k - 1 } ^ { i } ) ^ { - 1 } } \\
{ v _ { k } ^ { i } = u _ { k - 1 } ^ { i } ( v _ { k - 1 } ^ { i } ) ^ { b _ { k } } } \\
{ w _ { k } ^ { i } = w _ { k - 1 } ^ { i } }
\end{array} \left\{\begin{array} { l } 
{ u _ { k } ^ { 1 } = u _ { k } ^ { 2 } ( w _ { k } ^ { 2 } ) ^ { - \mu _ { k } } } \\
{ v _ { k } ^ { 1 } = v _ { k } ^ { 2 } ( w _ { k } ^ { 2 } ) ^ { \mu _ { k + 1 } } } \\
{ w _ { k } ^ { 1 } = ( w _ { k } ^ { 2 } ) ^ { - 1 } }
\end{array} \left\{\begin{array}{l}
u_{1}^{i}=\left(u^{0}\right)^{-1} \\
v_{1}^{i}=v^{0}\left(u^{0}\right)^{b_{1}} \\
w_{1}^{i}=w^{0}\left(u^{0}\right)^{-1},
\end{array}\right.\right.\right.
$$

where $\lambda_{k}, \mu_{k}$ and $b_{k}$ are given by (9).

Proof. Set $h=f_{2}, \cdots, f_{d}: \tilde{X} \rightarrow X_{1}$ and $W_{i}=h^{-1}\left(U_{i}\right)$ for $i=1,2,3$. Then $\left.h\right|_{W_{2} \cup W_{3}}$ defines the minimal resolution of $U_{2} \cup U_{3}$. Hence $W_{2} \cup W_{3}$ are covered by $2 s$ copies $V_{k}^{i}, 1 \leqq k \leqq s, i=1,2$ of $C^{3}$ with the coordinates $\left(u_{k}^{i}, v_{k}^{i}, w_{k}^{i}\right)$ and the transition functions among them are given by (14), namely,

$$
\left\{\begin{array} { l } 
{ u _ { k } ^ { i } = ( v _ { k - 1 } ^ { i } ) ^ { - 1 } } \\
{ v _ { k } ^ { i } = ( v _ { k - 1 } ^ { i } ) ^ { b _ { k } ^ { \prime } } u _ { k - 1 } ^ { i } } \\
{ w _ { k } ^ { i } = w _ { k - 1 } ^ { i } }
\end{array} \left\{\begin{array}{l}
u_{k}^{1}=u_{k}^{2}\left(w_{k}^{2}\right)^{-\left(\dot{\lambda}_{k}^{\prime}+n \mu_{k}^{\prime}\right) / p} \\
v_{k}^{1}=v_{k}^{2}\left(w_{k}^{2}\right)^{\left(\lambda_{k+1}^{\prime}+n \mu_{k+1}^{\prime}\right) / p} \\
w_{k}^{1}=\left(w_{k}^{2}\right)^{-1}
\end{array}\right.\right.
$$

where $\lambda_{k}^{\prime}, \mu_{k}^{\prime}, b_{k}^{\prime}$ are determined by the euclidian algorithm of (9) putting this time $\lambda_{0}=p$ and $\lambda_{1}=p_{2}$. In fact, in this case we can take $a_{1}=-1 / p$ and $a_{2}=n / p$ in (14), as is seen from (12). But if $\lambda_{k}$ and $\mu_{k}$ are the integers defined by (9) from $n$ and $p$, then we can show inductively the following relations;

$$
\lambda_{k}^{\prime}=\lambda_{k+1}, b_{k}^{\prime}=b_{k+1} \quad(0 \leqq k \leqq s-1), \quad \lambda_{k+1}+n \mu_{n}^{\prime}=p \mu_{k+1} \quad(k \geqq 1) .
$$

Hence we have obtained the first two relations. The last one is easily 
deduced from (12), (13) and (8).

Q.E.D.

Lemma 6. Suppose $f: \tilde{X} \rightarrow X$ and $f^{\prime}: \tilde{X}^{\prime} \rightarrow X$ be the two canonical resolutions of $X$, according to the isomorphisms $X \cong N_{n ; p, p}$ and $X \cong$ $N_{n ; 1, p^{\prime}}$ respectively, where $p$ and $p^{\prime}$ are related by $p p^{\prime} \equiv 1 \bmod n$. Then there exists an isomorphism $h: X \rightarrow X^{\prime}$ such that $f^{\prime} h=f$.

Proof. We shall only indicate the method of proof and leave the explicit computations to the readers. It suffices to prove that $\tilde{X}^{\prime}$ is covered by $2 s+1$ copies of $\boldsymbol{C}^{3}$ and that the transition functions between them are given by (15). Decompose $f$ into $f=f_{1} \cdots f_{d}$ as in Remark 3 . We prove the lemma by induction on $d$. In fact, in this case we have $U_{1} \cong U_{2} \cong \mathbb{C}^{3}$ and $U_{3}=N_{p^{\prime} ; 1, p_{3}^{\prime}}$ by (11). Thus we may apply the induction hypothesis to the canonical resolution $h: h^{-1}\left(U_{3}\right) \rightarrow U_{3}$, where $h=f_{2} \cdots f_{d} \mid\left(f_{d} \cdots f_{2}\right)^{-1}\left(U_{3}\right)$. Hence if we set $W_{3}=h^{-1}\left(U_{3}\right)$, then $W_{3}$ is isomorphic to the canonical resolution $\tilde{N}_{p^{\prime} ; p^{\prime \prime} p^{\prime \prime}}$ of $N_{p^{\prime} ; p^{\prime \prime} p^{\prime \prime}}$, where $p^{\prime \prime}$ is defined by the formula $p^{\prime \prime} p^{\prime} \equiv 1\left(\bmod p^{\prime}\right)$ and $0 \leqq p^{\prime \prime}<p^{\prime}$. Then we have to show that $\tilde{N}_{p^{\prime} ; p^{\prime \prime} p^{\prime \prime}}$ is isomorphic to $\bigcup_{k=1}^{s-1} V_{k}^{i} \cup V_{0}$ in the notation of Lemma 5. But this corresponds to the fact that if $n / p=$ $b_{1}-1 / b_{2}-1 / b_{3}-\cdots-1 / b_{s}, b_{i} \geqq 2$, is the expansion of $n / p$ into the continued fraction, then that of $p^{\prime} / p^{\prime \prime}$ is given by $p^{\prime} / p^{\prime \prime}=b_{1-1} 1 / b_{2}-\cdots_{-} 1 / b_{s-1}$. This can be derived from $n / p^{\prime}=b_{s-} 1 / b_{s-1} \cdots_{-} 1 / b_{1}$. Now it remains to see that the transition functions with respect to $V_{1}^{i}$ and $U_{0}$. But these can be calculated using (12) and (8) to coincide with the last relations of (15).

Q.E.D.

Now we define nonsingular subvarieties $\theta_{k}, 1 \leqq k \leqq s$, of $\tilde{X}$ by the following formula;

$$
\begin{aligned}
& \theta_{1}: u^{0}=0 \text { in } V_{0}, v_{1}^{1}=0 \text { in } V_{1}^{1}, v_{1}^{2}=0 \text { in } V_{1}^{2} \\
& \theta_{k}: \begin{cases}u_{k-1}^{i}=0 & \text { in } V_{k-1}^{i} \\
v_{k}^{i}=0 & \text { in } V_{k}^{i}\end{cases}
\end{aligned}
$$

Then $f^{-1}(P)=\bigcup_{k=1}^{s} \theta_{k}$, where $P$ is the singular point of $X$ and $\theta_{k}$ is the proper transform of the exceptional locus of $f_{k}$, when we decompose 
$f$ into $f=f_{1} \cdots f_{d}$ as usual. Note on the other hand that if $f^{\prime}=f_{1}^{\prime} \cdots f_{d}^{\prime}$ is the decomposition of $f^{\prime}$, then $\theta_{k}$ is the proper transform the exceptional locus of $f_{d-k}$. Roughly speaking, $f$ and $f^{\prime}$ are the resolutions of $X$ from the opposite sides. Now from Lemma 5 we can see immediately

Corollary to Lemma 6 [12]. i) $\theta_{1} \cong \boldsymbol{P}^{2}$ and $N_{\theta_{1} / \tilde{X}} \cong-b_{1} H$. ii) $\theta_{k}, k=2, \cdots, s$, is isomorphic to $\Sigma_{\mu_{k}}$. If $F$ is the general fibering of $\theta_{k}$, then $\left.N_{\theta_{k} / \tilde{X}}\right|_{F} \cong-b_{k} H$.

For the precise proof we refer the reader to Lemma 4.3-4.6 of [12]. We only note that since $\mu_{k} \geqq \mu_{2} \geqq 2$ for $k \geqq 2$, we conclude that $\theta_{k} \nsucceq \Sigma_{0}, \npreceq \Sigma_{1}$ for any $k$. Hence in particular there exists no exceptional surface of the first kind at all among $\theta_{k}$.

Now we continue the proof of the proposition. By virtue of the above results we may assume now that $\operatorname{dim} D_{i}=1$. Then $\widetilde{E}_{i}$ coincides with an irreducible component of $\left(f_{b} \cdots f_{d}\right)^{-1}\left(C_{j}\right)$ for some $C_{j}$. Set $f^{b}=f_{b} \cdots f_{d}$. Then $f^{b} \mid \widetilde{E}_{i}: \widetilde{E}_{i} \rightarrow C_{j}$ gives the natural structure of a $\boldsymbol{P}^{1}$ bunddle on $\widetilde{E}_{i}$. Then by [14] we see that $\left.N_{\tilde{E}_{i} / \tilde{X}}\right|_{F} \cong-b H_{F}$ with $b \geqq 2$, where $F$ is the general fiber of $f^{p} \mid \widetilde{E}_{i}$. Thus $\widetilde{E}_{i}$ can possibly be an exceptional surface of the first kind only along the incompatible fibering of $\widetilde{E}_{i}$. This proves the proposition.

Remark 5. If $n, p, q$ satisfy the following condition, then in the canonical resolution $f: \tilde{X} \rightarrow X$, the exceptional case of the proposition occurs:

Set $(p, q)=d$ and define integers $p_{i}, q_{j}, n_{i}^{p}, n_{j}^{q}, 0 \leqq i \leqq s+1 \quad 0 \leqq j \leqq t \leqq+1$, by the formulas;

$$
\begin{aligned}
& q=b_{0} p+p_{1}, 0 \leqq p_{1}<p, p=c_{0} q+q_{1}, 0 \leqq q_{1}<q \\
& p_{i-1}=b_{i} p_{i}-p_{i+1}, 0 \leqq p_{i+1}<p_{i}, q_{j-1} c_{j} q_{j}-q_{j+1}, 0 \leqq q_{j+1}<q_{j} \\
& p_{0}=p, p_{s}=d, p_{s+1}=0 \quad q_{0}=q, q_{t}=d, q_{p+1}=0 \\
& n=d_{0} p-n_{1}^{p}, 0 \leqq n_{1}^{p}<p \quad n=e_{0} q-n_{1}^{q}, 0 \leqq n_{1}^{q}<q \\
& n_{i-1}^{p}=d_{i} p_{i}+n_{i}^{p}, 0 \leqq n_{i}^{p}<p_{i}, \quad n_{j-1}^{q}=e_{j} q_{j}+n_{j}^{q}, 0 \leqq n_{q}<q_{j} .
\end{aligned}
$$


Then $d_{1}=n_{s+1}^{q p}=n_{t+1}^{q}$ is the integer defined by $d_{1}+n \equiv 0(\bmod d)$ and $0 \leqq d_{1}<d$. Now let $p_{i}^{\prime}=p_{i} / d, q_{j}^{i}=q_{j} / d$ and set

$$
\begin{array}{ll}
P^{\prime}=1 / p_{0}^{\prime} p_{1}^{\prime}+\cdots+1 / p_{s-1}^{\prime} p_{s}^{\prime}, & Q^{\prime}=1 / q_{0}^{\prime} q_{1}^{\prime}+\cdots+1 / q_{t-1}^{\prime} q_{i}^{\prime} \\
N_{p}^{\prime}=n_{1}^{p} / p_{0}^{\prime} p_{1}^{\prime}+\cdots+n_{s}^{p} / p_{s-1}^{\prime} p_{s}^{\prime}, & N_{q}^{\prime}=n_{1}^{q} / q_{0}^{\prime} q_{1}^{\prime}+\cdots+n_{l}^{q} / q_{t-1}^{\prime} q_{t}^{\prime} .
\end{array}
$$

Moreover let $\lambda_{k}, \mu_{k}, 1 \leqq k \leqq b$, be defined by the algorithm of (9) putting $\lambda_{0}=d$ and $\lambda_{1}=d_{1}$ there. Now our condition is stated as follows; there exists $k, 1 \leqq k \leqq b$, such that

$$
P^{\prime}+Q^{\prime}-1 / p^{\prime} q^{\prime}=\mu_{k} \quad \text { and } \quad N_{p}^{\prime}+N_{q}^{\prime}+1 / p^{\prime} q^{\prime}=\lambda_{k} .
$$

Remark 6. Lemma 6 and Proposition 1 would certainly be true for any special resolution.

As an example of the explicit resolutions we take $X=C^{3} / G$, where $G=\{g\}$ and $g$ acts on $\boldsymbol{C}^{3}$ by $g=\left(e_{5}, e_{5}^{2}, e_{5}^{3}\right)$. Then according to whether we take $g, g^{2}$, or $g^{3}$ as a generator of $G$, we have the isomorphisms $X \cong N_{5 ; 2,3}, X \cong N_{5 ; 2,4}$, and $X \cong N_{5 ; 3,4}$ respectively. Let $f_{i}: \tilde{X} \rightarrow X, 1 \leqq$ $i \leqq 3$, be the corresponding canonical resolutions of $X$. Then $f_{i}^{-1}(p)=$ $\theta_{(i)}$ look as in the following figure, where $P$ is the singular point of $X$.

$\mathrm{N}_{5} ; 23$

$\mathrm{N}_{5} ; 24$

$\theta_{(1)}$

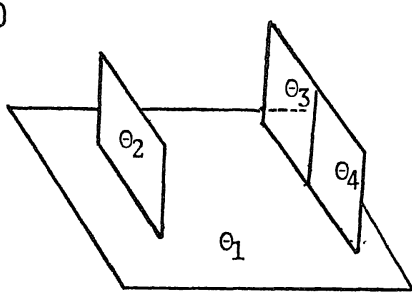

${ }^{\theta}(2)$

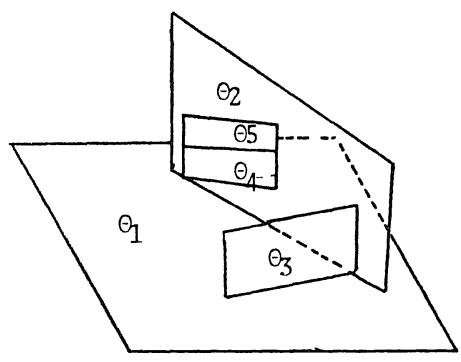

$\theta_{1}:$ rational

$\theta_{1} \cong \Sigma_{2}$

$\left.N_{\theta_{1} / \tilde{X}_{2}}\right|_{F} \cong-3 H_{F}$

$\theta_{2} \cong \boldsymbol{P}^{2} \quad N_{0_{2} / \tilde{X}_{1}} \cong-2 H$

$\left.\theta_{3} \cong \Sigma_{2} \quad N_{\theta_{3} / \bar{X}_{1}}\right|_{F} \cong-2 H_{F} \quad \theta_{2}:$ rational

$\theta_{4} \cong \boldsymbol{P}^{2} \quad N_{\theta_{4} / \tilde{X}_{1}} \cong-\left.2 H \quad \theta_{3} \cong \Sigma_{2} \quad N_{\theta_{3} / \tilde{X}_{2}}\right|_{F} \cong-2 H_{F}$

$\theta_{4} \cong \boldsymbol{P}^{2} \quad N_{\theta_{4} / \bar{X}_{2}} \cong-2 H$

$\left.\theta_{5} \cong \Sigma_{2} \quad N_{\theta_{5} / \bar{X}_{2}}\right|_{F} \cong-2 H_{E}$ 


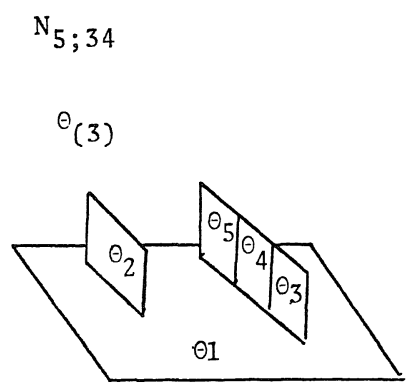

$$
\begin{array}{ll}
\theta_{1}: \text { rational } & \\
\theta_{2} \cong \boldsymbol{P}^{2} & N_{\theta_{2} / \tilde{X}_{3}} \cong-3 H \\
\theta_{3} \cong \Sigma_{2} & \left.N_{\theta_{3} / \tilde{X}_{3}}\right|_{F} \cong-2 H_{F} \\
\theta_{4} \cong \Sigma_{3} & \left.N_{\theta_{4} / \tilde{X}_{3}}\right|_{F} \cong-2 H_{F} \\
\theta_{5} \cong \boldsymbol{P}^{2} & N_{\theta_{5} / \tilde{X}_{3}} \cong-2 H
\end{array}
$$

\section{§2. Resolutions of General Isolated Quotient Singularities and Isolated Singularities with $\mathbb{C}^{*}$-action of Dimension 3}

2.1. Let $X$ be a complex space of dimension 3 and $\mathfrak{P}$ be a point of $X$. Suppose there exists a neighborhood $U$ of $\mathfrak{P}$ in $X$ such that $U$ is siomorphic to $D \times Y$, where $D$ is a unit disc $\{t ; 0 \leqq|t|<1\}$ and $Y$ is some neighborhood of the singular point $\mathfrak{Q}$ of the cyclic quotient singularity $N_{n, 1, p}$. Let $\varphi: U \cong D \times Y$ be the isomorphism, and $h: \widetilde{Y} \rightarrow Y$ be the minimal resolution of $Y$. Then $f=(\varphi)^{-1}\left(i d_{D} \times h\right): D \times \tilde{Y} \rightarrow U$ defines a resolution of $U$. Now let $U^{\prime}$ be another neighborhood of $\mathfrak{B}$ with an isomorphism $\varphi^{\prime}: U^{\prime} \cong D \times Y^{\prime}$ and let $f^{\prime} ; D \times \tilde{Y}^{\prime} \rightarrow U^{\prime}$ be the resolution obtained analogously using the minimal resolution $h^{\prime}: \tilde{Y}^{\prime} \rightarrow$ $Y^{\prime}$ of $Y^{\prime}$, where $Y^{\prime}$ is again some neighborhood of the singular point $\mathfrak{Q}^{\prime}$ of a cyclic quotient singularity $N_{n^{\prime}, 1, p^{\prime}}$. Then we have necessarily that $n=n^{\prime}$ and $p=p^{\prime 1)}$.

Lemma 7. The above $f$ and $f^{\prime}$ coincide on $U \cap U^{\prime}$, or more precisely, there exists an isomorphism $\psi: f^{-1}\left(U \cap U^{\prime}\right)=f^{\prime-1}\left(U \cap U^{\prime}\right)$ such that $f^{\prime} \psi=f$.

Proof. First, recall that a quotient singularity is ratinoal [1, Satz 1.7]. Then, by [7, Chap. I], [11, Theorem 1] any of its resolutions is obtained by succession of a finite number of quadratic transformations. Namely, let $Y_{0}$ be a quotient singularity and $h_{0}: \tilde{Y}_{0} \rightarrow Y_{0}$ a resolution.

1) This is in fact the consequence of the following lemma 7, which makes no use of this fact, together with the uniqueness of the minimal resolution of a normal singularity of a surface and the rigidity of quotient singularities [1]. 
Then $h_{0}$ can be written as $h_{0}=h_{d}^{0} \ldots h_{1}^{0}$, where $h_{i}^{0}: Z_{i} \rightarrow Z_{i-1}, 1 \leqq i \leqq d$, is the moniodal transformation with center the singular locus $S_{i}$ of $Z_{i}$ with its reduced structure and $Z_{0}=Y_{0}$ and $Z_{d}=\tilde{Y}_{0}$. In fact, $Z_{i}$ are all normal and $S_{i}$ consists only of finite number of points [7, Prop. 8.1], [11, Prop. 1.2]. Now we define a resolution $f_{0}: \tilde{U}^{\prime \prime} \rightarrow U^{\prime \prime}$ of $U^{\prime \prime}=U \cap U^{\prime}$ inductively as a succession of monoidal transformations with nonsingular center as follows; first let $f_{1}^{0}: U_{1} \rightarrow U^{\prime \prime}$ be the moniodal transformation with center the singular locus $S^{\prime \prime}$ of $U^{\prime \prime}$ ( $S^{\prime \prime}$ coincides with $U^{\prime} \cap \varphi(D \times$ $Q$ ) and hence is non-singular). Assume now that $f_{i}^{0}: U_{i} \rightarrow U_{k-1}, 1 \leqq i \leqq s$, have already be defined. Then we define $f_{s+1}^{0}: U_{s+1} \rightarrow U_{s}$ as a monoidal transformation with center the singular locus of $U_{s}$. Then by the definition of $f_{0}, f_{i}^{0}$ is naturally isomorphic to both $f_{i}=i d_{D} \times h_{i}: D \times Z_{i} \rightarrow D \times$ $Z_{l-1}$, and $f_{1}^{\prime}=i d_{D} \times h_{i}^{\prime}: D \times Z_{i}^{\prime} \rightarrow D \times Z_{i-1}^{\prime}$, where $h=h_{d} \ldots h_{1}$ with $h_{i}$ : $Z_{i} \rightarrow Z_{i-1} \quad$ (resp. $h^{\prime}=h_{d^{\prime}}^{\prime} \ldots h_{1}^{\prime}$ with $h_{i}^{\prime}: Z_{i}^{\prime} \rightarrow Z_{i-1}^{\prime}$ ) is the decomposition of $h$ (resp. $h^{\prime}$ ) into the quadratic transformations as above. Hence we see that $d=d^{\prime}, f^{0}=f_{d}^{0} . . f_{1}^{0}$ gives a resolution of $U \cap U^{\prime}$, and finally both $f$ and $f^{\prime}$ coincide with $f^{0}$ on $U \cap U^{\prime}$. This completes the proof.

We call the resolution obtained in the lemma the minimal resolution of $X$ at $\mathfrak{B}$. Moreover, suppose $X_{1}$ is a complex space of $\operatorname{dim} X_{1}=3$ and each point $\mathfrak{P}_{1} \in X_{1}$ admits a neighborhood $U_{1}$ which is isomorphic to $D \times Y_{1}$ with $Y_{1}$ a neighborhood of the singular point of some cyclic quotient singularity. Then a resolution $f: \tilde{X}_{1} \rightarrow X_{1}$ is said to be minimal if it gives the minimal resolution at each singular point of $X_{1}$.

2.2, Let $G \subseteq G L(3, \boldsymbol{C})$ be a finite subgroup. Then $G$ acts naturally on $\boldsymbol{C}^{3}$ and the quotient space $X=\boldsymbol{C}^{3} / G$ has the natural structure of a normal affine algebraic variety [10, Prop. 18]. Let $S$ be the singular locus of $X$. In this section we shall prove

Theorem 2. Suppose $X$ has only an isolated singularity at the point $\mathfrak{P}_{0}$ corresponding to the origin. Then there exists a resolution $f: \tilde{X} \rightarrow X$ of $X$ with the following propoerties; if we denote by $\theta_{1}, \ldots, \theta_{\text {s }}$ the irreducible components of $\theta=f^{-1}(S)$, then

1) $\theta$ has only normal crossings in $X$,

2) each $\theta_{i}$ is a nonsingular raitonal surface,

3) $\theta_{i} \cap \theta_{j}, i \neq j$, is isomprohic to $\boldsymbol{P}^{1}$ if it is not empty, and 
4) $\theta_{i} \cap \theta_{j} \cap \theta_{k}, i \neq j \neq k \neq i$ consists of a single point if it is not empty.

Proof. We follow after the proof of Satz 2.11 of [1]. We may assume that $G$ is small, namely, no elements of $G$ have 1 as its eigenvalues with multiplicity exactly 2 [9. Def.2]. Let $\sigma: W \rightarrow \mathbb{C}^{3}$ be the monoidal transformation at the origin. Then $E=\sigma^{-1}(0)$ is isomorphic to $\mathbb{C}^{3}-\{0\} / \mathbb{C}^{*}$, and hence to $\boldsymbol{P}^{2}$, where $\mathbb{C}^{*}$ acts naturally on $\mathbb{C}^{3}$. Further $W$ has the natural structure of a line bundle over $\boldsymbol{P}^{2}$. Let $\omega: W \rightarrow \mathbb{P}^{2}$ be the projection. The action of $G$ exetnds naturally onto $W$ so that it leaves $E$ invariant. In more detail, let $g \in G$ be an arbitrary element. There exists a linear change of coordinate of $\boldsymbol{C}^{3}$ such that with respect to this new coordinate $g$ has the diagonal form with eigenvalues $a, b$ and $c$ in this order. Let $\left(w_{1}, w_{2}, w_{3}\right)$ be this new coordinate. We may also consider $\left(w_{1}: w_{2}: w_{3}\right)$ as a homogeneous coordinate of $E \cong \boldsymbol{P}^{2}$. Now set $V_{i}=\left\{w^{i} \neq 0 ;(w) \in \boldsymbol{P}^{2}\right\}$. Then as usual $W$ is described as the union $W=\bigcup_{i=1}^{3}\left(V_{i} \times \boldsymbol{C}\right)$, where $\left(p, \zeta_{i}\right) \in V_{i} \times \boldsymbol{C}$ is identified with $\left(q, \zeta_{j}\right) \in V_{j} \times \mathbb{C}$ if and only if $\zeta_{i}=w_{i} / w_{j} \zeta_{j}$. Set $U_{i}=V_{i} \times \mathbb{C}$. Then $U_{i}$ is naturally isomorphic to $\boldsymbol{C}^{3}$ and the extended action of $g$ on $W$ is given with respect to this coordinate of $U_{i}$ by

$$
\begin{aligned}
g & =(b / a, c / a, a) \text { on } U_{1},=(a / b, c / b, b) \text { on } U_{2} \text {, and } \\
& =(a / c, b / c, c) \text { on } U_{3} .
\end{aligned}
$$

From this, we infer that the fixed point sets on $W$ of the elements of $G$ are classified according to the eigenvalues of the elements as follows; if the eigenvalues of an element $g \in G$ are as a set (i) $\{1, a, a\}$, (ii) $\{1, a, b\}$, (iii) $\{a, a, a\}$, (iv) $(a, a, b\}$ or (v) $\{a, b, c\}$, then the fixed point set of $g$ is (i) union of a fiber of $\omega$ and a line in $E$, (ii) union of a fiber and a point on $E$, (iii) $E$, (iv) uion of a line and a point on $E$, or (v) three distinct points in $E$, respectively, where $a, b$ and $c$ are roots of unity which are mutually distinct and different from 1 . Now let $X_{1}=W / G, \pi_{1}: W \rightarrow X_{1}$ the quotient map, and $f_{1}: X_{1} \rightarrow X$ be the morphism induced by $\sigma$. Note that $f_{1}$ is isomorphic outisde $f_{1}^{-1}\left(\mathfrak{B}^{0}\right)$ so that the singular locus $S_{1}$ of $X$ is contained in $f_{1}^{-1}\left(\mathfrak{P}^{0}\right)$. From this, we infer that no elements of $G$ can fix a fiber of $\omega$. Thus the classes (i) 
and (ii) above are empty. Now let $\mathfrak{B} \in E$ be an arbitray point, and $G_{\mathfrak{P}}$ be the stabilizer of $\mathfrak{P}$. We show that $G_{\mathfrak{P}}$ is cyclic. For this, we fix an open neighborhood $U$ of $\mathfrak{P}$ in $E$ such that $\omega^{-1}(U)$ is $G_{\mathfrak{P}}$ invariant. Such a $U$ exists because $G$ acts fiber-preservingly on $\omega: W \rightarrow \mathbb{P}^{2}$. Moreover, taking $U$ sufficiently small we may assume that $W \mid U$ is trivial i.e. there exists an isomorphism $\varphi_{U}: W \mid U \cong U \times \mathbb{C}$. Identify $W \mid U$ with $U \times \boldsymbol{C}$ by this isomorphism. Let $\zeta$ be the coordinate of $\boldsymbol{C}$. Then the action of an element $g$ of $G_{\mathfrak{3}}$ on the $\zeta$-component is of the following form $\zeta=h(u)_{b}^{c}$, where $h$ is a regular function on $U$. But since $g$ has a finite order, $h(u)$ is a root of unity and is a constant $a(g)$. Now define a map $\mu: G_{\Re \beta} \rightarrow C^{*}$ by $\mu(g)=a(g)$, then it is clear that $\mu$ is a homomorphism of the groups. It is easy to see that this $a(g)$ is independent of the trivialization $\varphi_{U}$ and coincides with the corresponding eigenvalue of $g$. Hence by the above classification of the elements of $G, \mu$ defines an isomorphism of $G_{\mathfrak{P}}$ with a subgroup of $\mathbb{C}^{*}$. Thus $G_{\mathfrak{P}}$ is cyclic. Now taking a generator $g$ of $G$ and a covering of $W$ by 3 open subsets $U_{1}, U_{2}$ and $U_{3}$ corresponding to $g$ as in the beginning of the proof, we see that $X_{1}$ is isomorphic at $\pi_{1}(\mathfrak{B})$ as a germ of a variety to a cyclic quotient singularity. Hence by Theorem 1 there exist a neighborhood $V$ of $\mathfrak{Q}=\pi_{1}(\mathfrak{P})$ in $X_{1}$ (in the usual topology), and a resolution $\left(\tilde{V}, f_{\mathfrak{B}}\right)$ of $V$ satifsying the properties stated in the Theorem. Moreover by the consideration in $1.4 f$ is minimal on $V-\mathfrak{Q}$. Finally, we take a finite number of points $\mathfrak{Q}_{1}, \ldots, \mathfrak{Q}_{s}$ of $X_{1}$, neighborhoods $U_{i}$ of $\mathfrak{Q}_{i}$ in $X_{1}$, and resolutions $f_{i}: \tilde{U}_{i} \rightarrow U_{i}$, such that $U_{i}$ cover the singular locus of $X_{1}$. Then by Lemma 7 we can see that these $f_{i}$ coincide on the intersections, and thus patch together to give a resolution $f_{2}: \widetilde{X} \rightarrow X_{1}$ of $X_{1}$. Then $f=f_{1} \cdot f_{2}: \tilde{X} \rightarrow X$ is the desired resolution. In fact, since any irreducible component of $S_{1}$ is either isomorphic to $P^{1}$ or a point, we deduce that each $\theta_{i}$ is nonsingular and rational except the proper transform, say $\theta_{1}$, of $f_{1}^{-1}\left(\mathfrak{P}^{0}\right)$ in $\tilde{X}$. But the latter is obtained by resolving the singularity of $f_{1}^{-1}\left(\mathfrak{P}^{0}\right)$, which in turn is a projective plane $E$ divided by $G$, considering $G$ as a subgroup of $\operatorname{PGL}(3, \mathbb{C})$. Hence by the theorem of Castelnuovo (See e.g. Šavarevič, Alg. Surfaces Steklov Institute of Math. 1965), $f_{1}^{-1}\left(\mathfrak{\beta}_{0}\right)$ and thus $\theta_{1}$ is rational. The other statements can be treated analogously and we do not repeat it, 
Remark 7. The above proof shows that also in the higher dimensional cases the stabilizer $G_{\mathfrak{B}}$ at each point $\mathfrak{P} \in E$ is cyclic if $X$ has only an isolated singularity. But if we allow $X$ to have the singularity of positive dimensions, then the map $\mu$ above has necessarily a kernel and $G_{\mathfrak{B}}$ is in general not cyclic.

2.3. Suppose $X$ is a normal affine algebraic variety embedded in $\boldsymbol{C}^{n}\left(z_{1}, \ldots, z_{n}\right)$ and there exists a $\mathbb{C}^{*}$ action $\mu$ on $\mathbb{C}^{n}$ which leaves $X$ invariant of the form;

$$
\mu\left(t,\left(z_{1}, \ldots, z_{n}\right)\right)=\left(t^{q_{1}} z_{1}, \ldots, t^{q_{n}} z_{n}\right), \quad t \in \mathbb{C}^{*},
$$

where $q_{i}$ are positive integers satisfying $\left(q_{1}, \ldots, q_{n}\right)=1[\mathrm{cf} .8]$. This means in particular that the action is effective. We assume further that $X$ is not contained in any linear subspace of $\mathbb{C}^{n}$. Noe suppose that $X$ has an isolated singularity at the origin. We call such a variety $X$ an isolated singularity with $C^{*}$ action. For such an $X$ we have the canonical way of inserting a '0-section' at the singular point, due to Orlik and Wagreich [8, 1.2]. More precisely, let $X^{\prime}=X-\{0\}, Z=X^{\prime} / \mathbb{C}^{*}, \pi^{\prime}$ : $X^{\prime} \rightarrow Z$ be the projection and $\Gamma^{\prime} \subseteq X^{\prime} \times Z$ be the graph of $\pi^{\prime}$. It is known that $Z$ is a projective variety [8].

Let $\Gamma$ be the closure of $\Gamma^{\prime}$ in $X \times Z$ and $\beta_{1}: \Gamma \rightarrow X(\operatorname{resp} \pi: \Gamma \rightarrow Z)$ be induced by the natural projection $p_{1}: X \times Z \rightarrow X$ (resp $p_{2}: X \times Z \rightarrow Z$ ) to the first (resp. to the second) factor. We have then the canonical section $i: Z \rightarrow \Gamma$ defined by $i(Z)=(0, z) \in X \times Z$. In the sequel we identify $Z$ with $i(Z)$. Then we show

Lemma 8. $\Gamma$ has only the cyclic quotient singularities: For each point $\overline{\mathfrak{P}} \in \Gamma, \Gamma$ is isomorphisc as a germ of an analytic space at $\overline{\mathfrak{P}}$ to a cyclic quotient singularity.

Proof. We consider $\Gamma$ and $Z$ as analytic spaces. First, by the fundamental result of Holmann [5], for each point $\mathfrak{B} \in \Gamma^{\prime}$ there exists a neighborhood $U_{\mathfrak{B}}$ of $\mathfrak{P}$ in $\Gamma^{\prime}$ and a 1-codimensional closed submanifold $A_{\mathfrak{P}}$ of $U_{\mathfrak{B}}$ such that (i) both $U_{\mathfrak{P}}$ and $A_{\mathfrak{P}}$ are $G_{\mathfrak{P}}$ invariant and (ii) $\pi^{\prime} \mid A_{\mathfrak{F}}$ induces an isomorphism between $A_{\mathfrak{B}} / G_{\mathfrak{B}}$ and some neighborhood $V_{\overline{\mathfrak{P}}}$ of $\overline{\mathfrak{P}}=\pi^{\prime}(\mathfrak{P})$, where $G_{\mathfrak{P}}$ is as usual the stabilizer of $\mathfrak{P}$ (refer [2] for 
the arguments). Now we fix a point $\overline{\mathfrak{P}} \in Z$ and then a point $\mathfrak{B} \in$ $\pi^{-1}(\overline{\mathfrak{P}})$ and take $U_{\mathfrak{\beta}}, A_{\mathfrak{B}}$ and $V_{\overline{\mathfrak{P}}}$ as above. Let $Y=\pi^{\prime-1}\left(V_{\overline{\mathfrak{P}}}\right)$. Define a holomorphic retraction $r: Y \rightarrow \pi^{\prime-1}(\overline{\mathfrak{P}})$, as follows; let $\mathfrak{Q} \in Y$ be any point. Then there exists a $t \in C^{*}$ such that $\mu(t, \mathfrak{Q}) \in A_{\mathfrak{1}}$. Then we define $r(\mathfrak{Q})=\mu(t, \mathfrak{P})$. This is easily seen to be independent of the choice of such a $t$, and the map is well-defined. Note that $\pi^{\prime-1}(\overline{\mathfrak{P}})$ is naturally isomorphic to $C^{*} / G_{\mathfrak{P}}$. Then we set $\tilde{Y}=Y \times_{C^{*} / G_{\mathfrak{B}}} \mathbb{C}^{*}$, the fiber product of $Y$ and $\boldsymbol{C}^{*}$ over $\pi^{\prime-1}(\overline{\mathfrak{P}})=\boldsymbol{C}^{*} / G_{\mathfrak{\beta}}$, where $\mathbb{C}^{*} \rightarrow \mathbb{C}^{*} / G_{\mathfrak{\beta}}$, which we denote by $\alpha$, is the natural projection. Then there exists an isomorphism $\varphi: \tilde{Y} \rightarrow C^{*} \times A_{\mathfrak{B}}$ and the following diagram is commutative;

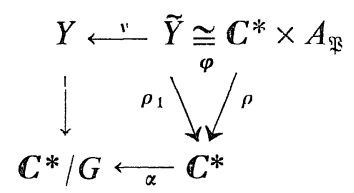

where $v$ and $\rho$ are natural projections and $\rho_{1}$ the projections to the first factor. Indeed, $\varphi$ is explicitly defined by $\varphi^{-1}(t, a)=(t, \mu(t, a))$, with $t \in \mathbb{C}^{*}$ and $a \in A_{\mathfrak{s}}$.

Further, the natural actions of $G_{\mathfrak{P}}$ on $\tilde{Y}$ and $\mathbb{C}^{*} \times A_{\mathfrak{B}}$ commute with $\varphi$ and thus $Y=\tilde{Y} / G_{\mathfrak{F}}$ is isomorphic to $\left(\mathbb{C}^{*} \times A_{\mathfrak{P}}\right) / G_{\mathfrak{P}}$. On the other hand $G_{\Re}$ acts naturally on $\boldsymbol{C} \times A_{\mathfrak{P}}$ and $\boldsymbol{C}$, extending those on $\boldsymbol{C}^{*} \times A_{\mathfrak{\beta}}$ and $C^{*}$ respectively. From this we infer that $\pi^{-1}\left(V_{\overline{\mathfrak{B}}}\right) \cong\left(\mathbb{C} \times A_{\mathfrak{B}}\right) / G_{\mathfrak{P}}$ with respect to this action of $G_{\mathfrak{B}}$ on $C \times A_{\mathfrak{B}}$. This proves the lemma, since $G_{\mathfrak{P}}$ is cyclic.

Remark 8. Acutually, we have the following commutative diagram extending the above one;

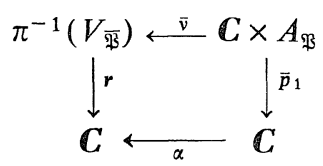

such that the map $\bar{v}$ is equavariant, where $\mathbb{C}^{*}$ acts on $\mathbb{C} \times A_{\mathfrak{B}}$ in a natural manner.

Combining Lemma 7 and Lemma 8 with Theorem 1 we have the following theorem when dimension $X=3$. 
Theorem 3. Suppose $X$ is an isolated singularity with $\mathbb{C}^{*}$ action and $\operatorname{dim} X=3$. Then there exists an equivariant resolution $f: \tilde{X} \rightarrow X$ of $X$ with the following properites; if we denote by $\theta_{0}, \ldots, \theta_{b}$, the irreducible components of $\theta=f^{-1}(\mathfrak{B})$ with $\mathfrak{P}_{0}$ the singular point of $X$, then

1) $\theta$ has only normal crossings in $\tilde{X}$.

2) each $\theta_{i}$ is a nonsingular ruled surfce (i.e. it is birationally equivalent to the product of a nonsingular curve $C$ and projective line $\left.\mathbb{P}^{1}\right)$ except one, say $\theta_{0}$, which is the proper transform of $i(Z) . \theta_{0}$ also is nonsingular.

3) $\theta_{i} \cap \theta_{j}, i \neq j$ is a nonsingular curce.

We only note that the equivariance can be deduced from the above remark.

It may be conjectured that the same kind of resolution could be obtained also in higher dimensional cases.

As an example we shall examine the resolutions of Brieskorn varieties. Let $X_{a}$ be a hypersurface in $\mathbb{C}^{4}$ defined by the equation

$$
z_{1}^{a_{1}}+z_{2}^{a_{2}}+z_{3}^{a_{3}}+z_{4}^{a_{4}}=0
$$

where $a_{i}$ are integers $\geqq 2$. Put $a=l$.c. $d$. of $a_{i}$ and $q_{i}=a / a_{i}$. Then the map $h: \mathbb{C}^{4}(t) \rightarrow \mathbb{C}^{4}(z)$ with $f\left(t_{1}, t_{2}, t_{3}, t_{4}\right)=\left(t_{1}^{q_{1}}, t_{2}^{q_{2}}, t_{3}^{q_{3}}, t_{4}^{q_{4}}\right)$ defines an abelian covering $X_{1}$ of $X_{a}$ in $\mathbb{C}^{4}(t)$. In fact $X_{1}$ is defined by

$$
t_{1}^{a}+t_{2}^{a}+t_{3}^{a}+t_{4}^{a}=0
$$

Let $f: L \rightarrow X_{1}$ be the monoidal transformation at the origin. $L$ had naturally the structure of a line bundle over a nonsingular surface $S$ defined by (16), considering this time $\left(t_{1}: t_{2} ; t_{3}: t_{4}\right)$ as a homogeneous coordinates of $\boldsymbol{P}^{3}$. The action of $G$ extends onto $L$ and the diagram

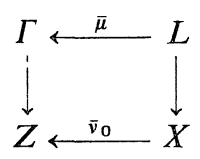

commutes and compatible with the natural $\mathbb{C}^{*}$ action on both $L$ and $\Gamma$, where $\Gamma$ and $Z$ are as above. Now let $H_{i}$ and $l_{i j}$ be the hyperplanes and lines in $\mathbb{P}^{2}$ defined by $t_{i}=0$, and $t_{i}=t_{i}=0$ respectively. Then 
$C_{i}=H_{i} \cap X$ is a nonsingular curve and $l_{i j} \cap X$ consists of $n$ distinct points $\mathfrak{P}_{i j}^{(1)}, \ldots, \mathfrak{P}_{i j}^{(\mu)}$. Hence we identified $X$ with the 0 -section of $L$. Then it is readily seen that $\Gamma$ has a singular point at $v\left(\mathfrak{P}_{i j}^{(\alpha)}\right)$, isomorphic as germs of anayltic spaces to the cyclic quotient singularity $\mathbb{C}^{3} /\left\{g_{i j}\right\}$, where $g_{i j}$ acts on $\mathbb{C}^{3}$ by

$$
g_{i j}=\left(e_{c_{k l}}, e_{c_{k l}}^{-q_{i}}, e_{c_{k l}}^{-q_{j}}\right),
$$

where $c_{k l}=\left(q_{k}, q_{l}\right)$ and $i, j, k, l$ are all distinct.

Here are some examples:

1) $\left(a_{i}, a_{j}\right)=1, i \neq j$. Then $g_{i j}=\left(e_{a_{i} a_{j}}, e_{a_{j}}^{-a_{k} a_{l}}, e_{a_{i}}^{-a_{k} a_{l}}\right)$. In this case $Z$ is a projective plane [2] and $v\left(C_{i}\right)$ form 4 lines in general position. Then using Lemma 3 we could resolve the singularity of $\Gamma$ rather easily. Note that then the irreducible components $\theta_{i}$ are all rational.

2) $a_{1}=a_{2}=b$ and $a_{3}=a_{4}=c$. Put $(b, c)=d, \quad b^{\prime}=b / d$ and $c^{\prime}=c / d$. In this case $\Gamma$ has $b$ singular points each isomorphic to $N_{c^{\prime} ; b_{1}^{\prime}, b_{1}^{\prime}}$ and $c$ singular points each isomorphic to $N_{b^{\prime} ; c_{1}^{\prime}, c_{1}^{\prime}}$ where $b_{1}^{\prime}$ and $c_{1}^{\prime}$ are determined by the following formula; $0 \leqq b_{1}^{\prime}<c^{\prime}, b_{1}^{\prime}+b^{\prime} \equiv 0(c) \quad 0 \leqq c_{1}^{\prime}<c^{\prime}$, $c_{1}^{\prime}+c^{\prime} \equiv 0\left(\bmod b^{\prime}\right)$. The resolution of each singular point is obtained according to Lemma 5 .

3) $a_{1}=b, a_{2}=a_{3}=a_{4}=c$. Let $d, b^{\prime}$ and $c^{\prime}$ be as above. $\Gamma$ has the singular locus along the curve $v\left(C_{1}\right)$, of the type $N_{b^{\prime} ; c_{1} 1}$. Let $f_{1}: \tilde{X} \rightarrow \Gamma$ the minimal resolution of $\Gamma, \theta_{0}$ the proper transform of $Z$ in $\tilde{X}$ and $\theta_{1}, \ldots, \theta_{s}$ be the irreducible components of $f_{1}^{-1}\left(v\left(c_{1}\right)\right)$ such that $\theta_{0} \cap \theta_{1}=\bar{C} \neq \phi$. Then $\theta_{0}$ is isomorphic to the cyclic covering of $\mathbb{P}^{2}$ of degree $d$, with branch locus $C$ defined by $z_{2}^{c}+z_{3}^{c}+z_{4}^{c}=0$, considering $\left(z_{2}: z_{3}: z_{4}\right)$ as the homogeneous coordinates of $\mathbb{P}^{2}$. Thus $\theta_{0}$ is isomorphic to $\boldsymbol{P}^{2}$ if $d=1$ and isomorphic to $\boldsymbol{P}^{1} \times \boldsymbol{P}^{1}$ if $d=2$. $\theta_{i}, i \geqq 1$, are isomorphic to the $\boldsymbol{P}^{1}$ bundles over $C$. Further we can see that the normal bundle $N_{\theta_{0} / \tilde{X}}$ of $\theta_{0}$ in $\tilde{X}$ is given by

$$
N_{\theta_{0} / \bar{X}}=-\left(c^{\prime} p^{\prime}+1\right) / b^{\prime} c^{\prime}[\bar{C}]
$$

with $0 \leqq p^{\prime}<b^{\prime}$ and $-\left(c^{\prime} p^{\prime}+1\right) \equiv 0 \bmod b^{\prime} \quad$ (cf. [4]). From this, we infer that $\theta_{0}$ is the exceptional surface of the first kind if and only if either 
$l=1$ and $c \mid b-1$, or $c=2$ and $b=2 m$ is even.

In the latter case we have a birational morphism $h: \tilde{X} \rightarrow X_{0}$ such that $X_{0}$ is nonsingular, $h$ is isomorphic outside $\theta$ and $C_{0}=h(\theta)$ is isomorphic to $\mathbb{P}^{1}$ and the normal bundle $N_{C_{0} / X_{0}}$ of $C_{0}$ in $X_{0}$ is isomorphic to $\left(-2 H_{C_{0}}\right) \oplus 1_{C_{0}}$, where $H_{C_{0}}$ and $1_{C_{0}}$ denotes the hyperplane bundle of $C_{0}$ and the trivial bundle respectively.

\section{References}

[1] Brieskorn, E., Rationale Singuiaritäten komplexer flächen, Invent. Math. 4 (1968), 336-358.

[2] Brieskorn, E. and Van de Ven, A., Some complex structures on products of homotopy spheres, Topology 7 (1968), 389-393.

[3] Hirzebruch, F., Über vierdimensionale Riemannsche Flächen mehrdetiger analytischer Funktionen von zwei komplexen Veräderlichen, Math. Ann. 126 (1953), 1-22.

[4] Hirzebruch, F. and Jänig, K., Involutions and singularities, Proceedings of the Bombay Colloq. on Alg. Geometry, Oxford (1969), 220-240.

[5] Holmann, H., Quotiennteräume komplexer Mannigfaltigkeiten nach komplexen Lieschen Automorphismengruppen, Math. Ann. 139 (1960), 383-402.

[6] Kempf, G., Knudsen, F., Mumford, D., and Saint-Donat, B., Toroidal embeddings I, Lecture Notes in Math., 339 Springer, (1973).

[7] Lipman, J., Rational singularities, with applications to algebraic surfaces and unique factorization, Publ. Math. IHES, 36 (1969), 195-279.

[8] Orlik, P. and Wagreich, P., Isolated singularities of algebraic surfaces with $C^{*}$ action, Ann. of Math. 93 (1971), 205-228.

[9] Prill, D., Local classification of quotients of complex manifolds by discontinuous groups, Duke Math. J. 34 (1967), 375-386.

[10] Serre, J-P., Groupes algébriques et corps de classes, Hermann, Paris, (1959).

[11] Tyurina, G. N., Absolute isolatedness of rational singularities and triple rational points, Functional analysis and its applications, 2 (1968), 324-333.

[12] Ueno, K., On fiber spaces of normally polarized abelian varieties of $\operatorname{dim} 2, \mathrm{I}, J$. Fac. Sci. Univ. of Tokyo, Sec. IA, 18 (1971), 37-95.

[13] Cartan, H., Quotient d'un espace analytique par un groupe d'automorphismes, in "algebraic geometry and topology", Princeton, (1957), 90-102. 